\title{
Performance Evaluation of the CHKED Multi-Elemental Analysis and Software
}

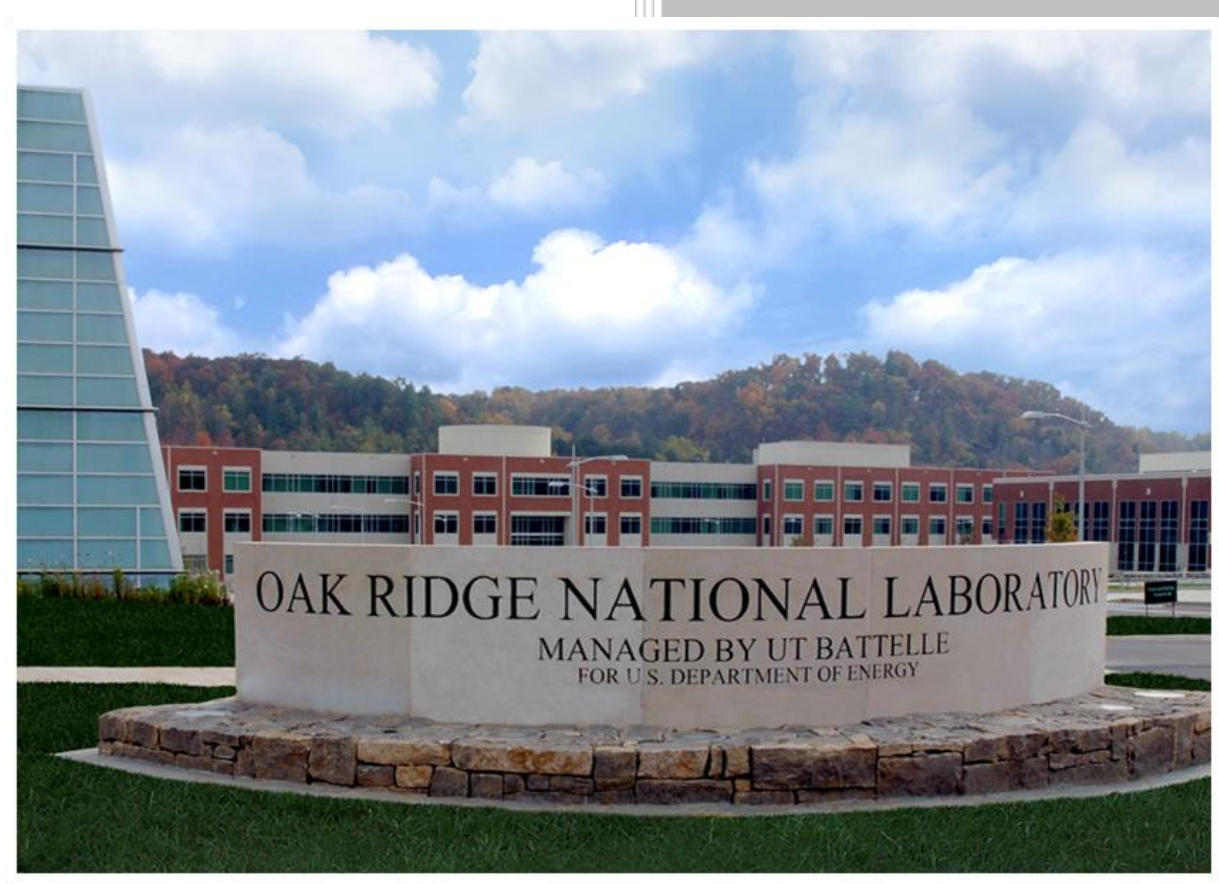

Robert D. McElroy

May 2018 


\title{
DOCUMENT AVAILABILITY
}

Reports produced after January 1, 1996, are generally available free via US Department of Energy (DOE) SciTech Connect.

Website www.osti.gov

Reports produced before January 1, 1996, may be purchased by members of the public from the following source:

\author{
National Technical Information Service \\ 5285 Port Royal Road \\ Springfield, VA 22161 \\ Telephone 703-605-6000 (1-800-553-6847) \\ TDD 703-487-4639 \\ Fax 703-605-6900 \\ E-mail info@ntis.gov \\ Website http://classic.ntis.gov/
}

Reports are available to DOE employees, DOE contractors, Energy Technology Data Exchange representatives, and International Nuclear Information System representatives from the following source:

Office of Scientific and Technical Information

PO Box 62

Oak Ridge, TN 37831

Telephone 865-576-8401

Fax 865-576-5728

E-mail reports@osti.gov

Website http://www.osti.gov/contact.html

This report was prepared as an account of work sponsored by an
agency of the United States Government. Neither the United States
Government nor any agency thereof, nor any of their employees, makes
any warranty, express or implied, or assumes any legal liability or
responsibility for the accuracy, completeness, or usefulness of any
information, apparatus, product, or process disclosed, or represents that
its use would not infringe privately owned rights. Reference herein to
any specific commercial product, process, or service by trade name,
trademark, manufacturer, or otherwise, does not necessarily constitute
or imply its endorsement, recommendation, or favoring by the United
States Government or any agency thereof. The views and opinions of
authors expressed herein do not necessarily state or reflect those of the
United States Government or any agency thereof.


Nuclear Security and Isotope Technology Division

\title{
PERFORMANCE EVALUATION OF THE CHKED MULTI-ELEMENTAL ANALYSIS
} AND SOFTWARE

\author{
Robert D. McElroy
}

Original Date Published: June 2016

Revision 1: May 2018

\author{
Prepared by \\ OAK RIDGE NATIONAL LABORATORY \\ Oak Ridge, TN 37831-6283 \\ managed by \\ UT-BATTELLE, LLC \\ for the \\ US DEPARTMENT OF ENERGY \\ under contract DE-AC05-00OR22725
}





\section{CONTENTS}

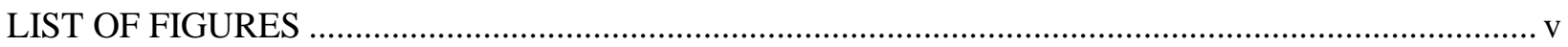

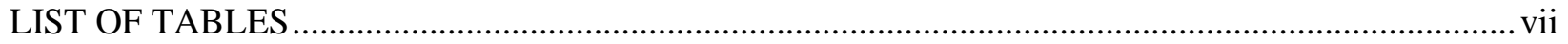

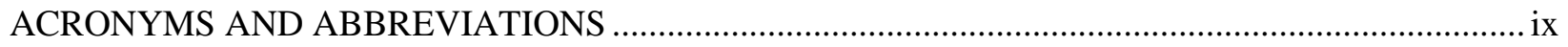

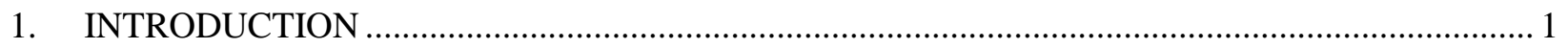

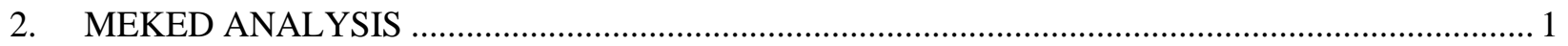

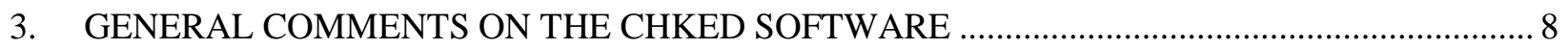

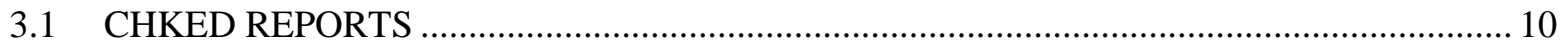

4. PERFORMANCE OF THE CHKED K-EDGE TRANSMISSION ANALYSIS METHODS .......... 10

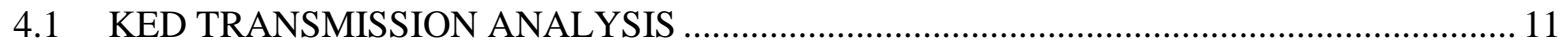

4.2 PERFORMANCE WITH PLUTONIUM AS THE PRIMARY ELEMENT ............................ 12

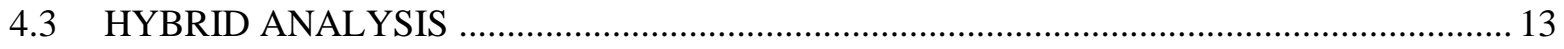

4.4 PERFORMANCE OF THE CHKED MEKED ANALYSIS …......................................... 15

4.5 IMPACT OF THE AM DECLARATION ON THE ASSAY RESULT . ............................ 16

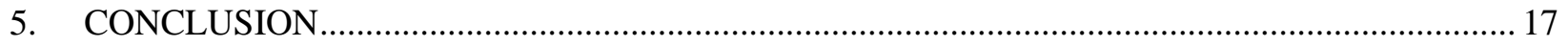

APPENDIX A

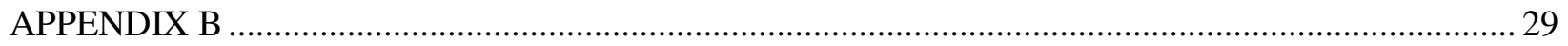

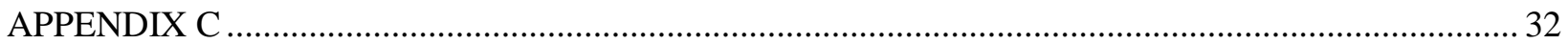

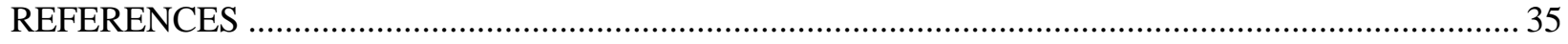





\section{LIST OF FIGURES}

Figure 1. MEKED default ROIs overlain on a portion of the KED spectrum from a U, Pu sample.

The ROIs tend to encroach into the high energy portion of the Gaussian-broadened K-

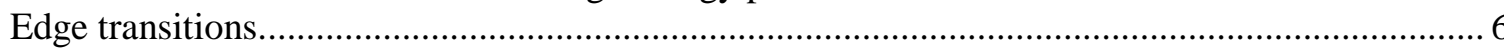

Figure 2. KED calibration curve for uranium-only solutions. ........................................................ 9

Figure 3. Plot of the ratio of measured-to-declared uranium concentration values for the ORNL

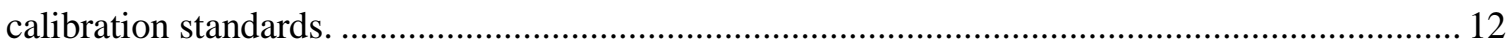

Figure 4. Peak shape from a Ge(Li) detector (from [5]) ............................................................... 19

Figure 5. Step background determined for a $321.9 \mathrm{~g} \mathrm{U} / \mathrm{L}$ solution using the VMS and CHKED

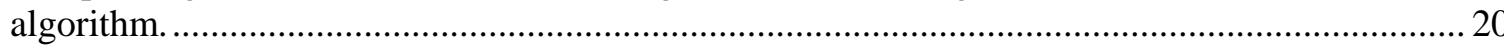

Figure 6. Plot showing the random coincidence summing contribution to the KED transmission

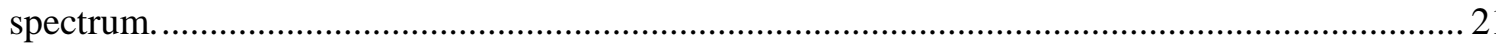

Figure 7. Plot of the passive (X-ray off) KED spectrum showing the contribution to the lower ROI from the ${ }^{109} \mathrm{Cd}$ source (acquisition time $=40,000 \mathrm{~s}$, peak rate $\sim 27 \mathrm{cps}$ ). ........................... 22

Figure 8. Measured KED transmission spectra illustrating the impact of the ${ }^{109} \mathrm{Cd}$ peak exponential tail on the lower background ROI.

Figure 9. Comparison of the MCNP6 simulation of a KED transmission measurement for a $270 \mathrm{~g}$

$\mathrm{U} / \mathrm{L}$ aqueous sample using corrected uranium photon cross-section libraries with a measured spectrum of the same concentration.

Figure 10. Low energy portion of the KED spectrum from a $321 \mathrm{~g} \mathrm{U} / \mathrm{L}$ sample without the ${ }^{109} \mathrm{Cd}$

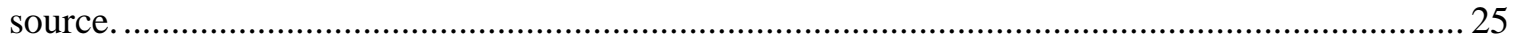

Figure 11. Plot illustrating the variation in the lower background ROI for the various ${ }^{109} \mathrm{Cd}$ source strengths.

Figure 12. Plot of the calculated step background beneath the uranium K-Edge transition at $115.6 \mathrm{keV}$ for a 1,000 second assay of the $321 \mathrm{~g} \mathrm{U} / \mathrm{L}$ sample for two different current settings of the $\mathrm{X}$-ray generator.

Figure 13. Relative reported U concentration of a $322 \mathrm{~g} \mathrm{U} / \mathrm{L}$ solution as a function of the ${ }^{109} \mathrm{Cd}$ peak count rate for the ORNL HKED system using an SGN vial with X-ray generator beam current of $15 \mathrm{~mA}$.

Figure 14. Relative reported concentration for a $322 \mathrm{~g} \mathrm{U} / \mathrm{L}$ sample as a function of time since last calibration assuming an initial Cd source rate of $420 \mathrm{cps}$.

Figure 15. Relative reported concentration for a $322 \mathrm{~g} \mathrm{U} / \mathrm{L}$ sample as a function of time since last calibration assuming two different initial Cd source rates with $10 \mathrm{~mA} \mathrm{X}$-ray generator beam current using the ORNL HKED system using the SGN vial............................................. 28

Figure 16. Sketch of the ORNL HKED system showing the approximate location within the shield housing of the thermal couple used for sample temperature measurement.

Figure 17. Concentration reported by the CHKED software as a function of temperature showing a temperature dependence of $+0.11 \% /{ }^{\circ} \mathrm{C}$.

Figure 18. Concentration reported by the CHKED software as a function of temperature after the temperature correction has been removed and exhibiting a temperature dependence of $+0.06 \% /{ }^{\circ} \mathrm{C}$.

Figure 19. Comparison of the uncorrected uranium concentration as a function of temperature analyzed using the VMS KED analysis and the CHKED analysis........................................... 31

Figure 20. Determination of the XRF-detectable interaction region by MCNP simulation. ..................... 33

Figure 21. Heat map of the XRF-detectable interaction region determined by MCNP simulation............ 33 



\section{LIST OF TABLES}

Table 1. Impact of the minor actinide declaration value on the assay MEKED assay result (the same spectra were analyzed using different declarations).

Table 2. K-Edge transition energies and $\mathrm{ROI}$ windows for $\mathrm{U}$ and $\mathrm{Pu}$................................................5

Table 3. Typical measurement precision for the CHKED KED assay for uranium solutions in the

SGN vial (1,000 s assay times).

Table 4. Typical measurement biases observed for two different HKED systems.....

Table 5. Measurement biases observed for the plutonium only solutions provided for this study.

Table 6. CHKED Hybrid mode analysis results for $\mathrm{U}: \mathrm{Pu}>10$.

Table 7. Comparison of CHKED Hybrid mode measured MOX sample assay result with declared values.

Table 8. Assay bias resulting from the CHKED Hybrid K-Edge analysis of the MOX spectra provided for this study.

Table 9. Assay bias resulting from the CHKED Hybrid K-Edge analysis of spectra provided from a reprocessing facility after recalibration.

Table 10. Comparison of MEKED measured MOX sample assay result with declared values after recalibration.

Table 11. Assay bias resulting from the CHKED MEKED analysis of spectra provided from a reprocessing facility after recalibration.

Table 12. Impact of the Am declaration value of the non-extrapolated assay result for two samples. 



\section{ACRONYMS AND ABBREVIATIONS}

$\begin{array}{ll}\text { CHKED } & \text { Canberra Hybrid K-Edge Software } \\ \text { DOE } & \text { US Department of Energy } \\ \text { FWHM } & \text { full width at half maximum } \\ \text { HKED } & \text { Hybrid K-Edge Densitometer } \\ \text { HPGe } & \text { high-purity germanium } \\ \text { HV } & \text { high voltage } \\ \text { IAEA } & \text { International Atomic Energy Agency } \\ \text { IDMS } & \text { Isotope Dilution Mass Spectroscopy } \\ \text { ISOCS } & \text { In Situ Object Counting System } \\ \text { KED } & \text { K-Edge densitometry } \\ \text { MCNP } & \text { Monte Carlo N-Particle } \\ \text { MEKED } & \text { Multi-elemental K-Edge } \\ \text { MOX } & \text { mixed oxide } \\ \text { ORNL } & \text { Oak Ridge National Laboratory } \\ \text { ROI } & \text { region of interest } \\ \text { VMS } & \text { (Virtual Memory System) Operating System } \\ \text { XRF } & \text { X-ray fluorescence }\end{array}$





\section{INTRODUCTION}

The Hybrid K-edge Densitometer (HKED) is a high precision safeguards assay system developed by Ottmar and Eberle [1] for quantification of the $\mathrm{U}$ and Pu concentrations of dissolver solutions in spent nuclear fuel reprocessing. The HKED measurement supplements traditional destructive assay methods such as Isotope Dilution Mass Spectrometry (IDMS) and is an essential component of the international safeguards program implementation at these facilities. The Canberra Hybrid K-Edge Densitometry (CHKED) software was recently developed by Canberra Industries to better address the changing composition of the nuclear fuel cycle by addition of a multi-elemental analysis capability to the K-Edge transmission measurement. Our evaluation of the CHKED software was performed at the request of the International Atomic Energy Agency (IAEA) to examine the functionality and performance of the CHKED software and algorithms and identify potential areas for improvement of the software. The reader is assumed to be familiar with the HKED technique and its current implementation in safeguards assay of $\mathrm{U}$ and $\mathrm{Pu}$ in the nuclear fuel cycle.

The Canberra Hybrid K-Edge Densitometry CHKED software replicates functionality of the older VMSbased Hybrid K-Edge Densitometer (HKED) software and incorporates additional analysis algorithms and tools. When the traditional HKED analyses are selected, the CHKED software appears to faithfully reproduce the measurement results as reported by the older software. The CHKED software includes a Multi-elemental K-Edge (MEKED) analysis based in part on algorithms devised at Los Alamos National Laboratory. In principle, the MEKED analysis represents a significant advancement over the traditional HKED analysis, providing a capability to analyze the actinide concentrations from complex solutions, such as low $\mathrm{U}: \mathrm{Pu}$ ratios (e.g., $\mathrm{U}: \mathrm{Pu}=1: 1$ ) including additional minor actinide components such as $\mathrm{Np}$ and Am. However, this MEKED analysis is somewhat limited by reliance on a region of interest (ROI) analysis of the spectrum, the need for extensive calibration measurements and a simplistic interpretation of the KED transmission spectrum. Finally, the MEKED algorithm set associated with the CHKED software (V1.0) acquired by Oak Ridge National Laboratory (ORNL) from Canberra Industries does not seem to be "finished" (e.g., the MEKED analysis seems to only determine measurement results for two actinides with while results for the minor actinides appear to be highly dependent on declaration values). These limitations are discussed in the sections below.

This report is an extensive revision of Reference [2]. Additions to the report include extended discussions on the impact of the ${ }^{109} \mathrm{Cd}$ normalization source on assay results, a detailed discussion of the performance of the CHKED algorithms (Section 4), and added discussions on temperature effects on the K-Edge densitometry (KED) transmission measurement (Appendix B) and sample vial dimensions (Appendix C).

\section{MEKED ANALYSIS}

The MEKED analysis expands the traditional ROI approach through the inclusion of additional ROIs corresponding to the various K-Edge transitions associated with the minor actinides. The traditional KED analysis assumes that only a single dominant actinide (typically $\mathrm{U}$ ) is present in the solution and that any other actinides are of sufficiently low concentration as to not perturb the interrogating X-ray flux. However, the presence of the actinides in the solution effectively alters the interrogating X-ray energy distribution compared to the assayed reference spectrum and introduces potentially large biases in the assay results unless this effect is properly accounted for in the analysis. The CHKED algorithms attempt to correct for the presence of additional actinides by introducing an attenuation matrix. 
The generalized MEKED approach involves the following steps ${ }^{1}$ :

1. Continuum background subtraction

2. Determine high voltage (HV) endpoint energies

3. First pass transmission determination and determination of current beam ratio $(\alpha)$

- Measured transmission ratios are determined rather than calculated from beam currents. A correction ratio is determined for each element. This has the benefit that it will tend to correct for variations in the energy distribution of the interrogating X-ray source (that is aging of the tube).

- An initial density is computed.

4. Second pass transmission determination and solve generalized MEKED equation

5. Enrichment correction

6. Temperature correction

Steps 1, 2, 5 and 6 follow the same methodology as the traditional KED transmission analysis. Biases associated with the continuum background subtraction are discussed in Appendix A. Steps 3 and 4 constitute the MEKED analysis enhancements.

\section{Generalized MEKED Equations}

The Canberra Hybrid K-Edge Densitometry User's Manual states that the elemental concentrations ( $\rho_{i}$, $\mathrm{g} / \mathrm{cm}^{3}$ ) are solved by the following densitometry equations:

$$
\left(\begin{array}{l}
\Delta \mu_{1,1} \Delta \mu_{1,2} \Delta \mu_{1,3} \Delta \mu_{1,4} \Delta \mu_{1,5} \Delta \mu_{1,6} \\
\Delta \mu_{2,1} \Delta \mu_{2,2} \Delta \mu_{2,3} \Delta \mu_{2,4} \Delta \mu_{2,5} \Delta \mu_{2,6} \\
\Delta \mu_{3,1} \Delta \mu_{3,2} \Delta \mu_{3,3} \Delta \mu_{3,4} \Delta \mu_{3,5} \Delta \mu_{3,6} \\
\Delta \mu_{4,1} \Delta \mu_{4,2} \Delta \mu_{4,3} \Delta \mu_{4,4} \Delta \mu_{4,5} \Delta \mu_{4,6} \\
\Delta \mu_{5,1} \Delta \mu_{5,2} \Delta \mu_{5,3} \Delta \mu_{5,4} \Delta \mu_{5,5} \Delta \mu_{5,6} \\
\Delta \mu_{6,1} \Delta \mu_{6,2} \Delta \mu_{6,3} \Delta \mu_{6,4} \Delta \mu_{6,5} \Delta \mu_{6,6}
\end{array}\right) \cdot\left(\begin{array}{l}
\rho_{1} \\
\rho_{2} \\
\rho_{3} \\
\rho_{4} \\
\rho_{5} \\
\rho_{6}
\end{array}\right)=\left(\begin{array}{l}
C_{1} \\
C_{2} \\
C_{3} \\
C_{4} \\
C_{5} \\
C_{6}
\end{array}\right)
$$

where $C_{j}=\frac{1}{x}\left[\exp \left(A_{j+}\right)-\exp \left(A_{j-}\right)\right]$,

$\mathrm{x}$ is the sample vial thickness

$\Delta \mu_{i, j}$ is the change in the mass attenuation coefficient across the K-edge jump evaluated from ROI to ROI

$A_{j \pm}=\ln (\ln (1 / T))$, at energy $E_{j \pm}$ bracketing the K-Edge energy of each major element (or ROI) $\mathrm{T}$ is the transmission through the sample.

$\underline{\text { Multi-elemental analysis limitations }}$

There are several small issues with the MEKED analysis

1. The MEKED is not truly multi-elemental.

Our studies (based on V1.0 software) indicate that the MEKED analysis is only applied to two elements. Data for "minor elements" can be entered into the declarations, but it is not clear from the documentation or the performance of the software how the minor elements are accounted for in the analysis.

\footnotetext{
${ }^{1}$ From Canberra CHKED training materials, presented by Marcel Villani at ORNL (2013).
} 


\section{$\underline{\text { Declarations and certificates }}$}

The CHKED MEKED software only determines concentration results for the three elements (two major plus one minor or one major plus two minor) listed in the declaration and certificate files. Although it is possible to assign calibration parameters for six elements (Th, $\mathrm{U}, \mathrm{Pu}, \mathrm{Np}, \mathrm{Am}$, and $\mathrm{Cm}$ ) using the CHKED software, it was only possible to assign three elements to a declaration or certificate. In the case of mixed oxide (MOX) items, this would typically mean assigning $U$ and $\mathrm{Pu}$, and then choosing $\mathrm{Am}$ or $\mathrm{Np}$ as the third element.

2. MEKED major element analysis considerations.

Since the software only allows definition of two major elements, the implication is that the multielemental analysis is only applied to two actinides and that the special case where there are only two major actinides is the only case addressed by this software. The Canberra Hybrid K-Edge Densitometry User's Manual defines this equation as

$$
\left(\begin{array}{l}
\Delta \mu_{1,1} \Delta \mu_{1,2} \\
\Delta \mu_{2,1} \Delta \mu_{2,2}
\end{array}\right) \cdot\left(\begin{array}{l}
\rho_{1} \\
\rho_{2}
\end{array}\right)=\left(\begin{array}{l}
C_{1} \\
C_{2}
\end{array}\right)
$$

The appeal of this representation is that it may be solved for the major actinide concentrations rather simply as set of two linear equations. However, it may be that the minor elements are included as known relative concentrations when applying the larger general attenuation matrix. We did not find definitive documentation to determine how the analysis is actually applied.

\section{Potential impact of the minor actinides on the major actinide results}

By examining the impact of the Pu content on the MEKED $U$ assay result we can estimate the impact of the minor elements on the $\mathrm{U}$ and $\mathrm{Pu}$. Typical delta-mu matrix values for $\mathrm{U}$ and $\mathrm{Pu}$ are

$$
\left(\begin{array}{l}
\Delta \mu_{1,1} \Delta \mu_{1,2} \\
\Delta \mu_{2,1} \Delta \mu_{2,2}
\end{array}\right)=\left(\begin{array}{cc}
3.53665 & -0.14022 \\
-0.04662 & 3.11345
\end{array}\right)
$$

Evaluating the two-element matrix with these attenuation values, we find that $\mathrm{Pu}$ in the solution begins to impact the $\mathrm{U}$ concentration when the Pu concentration exceeds $10 \mathrm{~g} / \mathrm{L}$ (at which point it should be considered a major actinide). Assuming a similar impact from the presence of small amounts of $\mathrm{Np}$ or $\mathrm{Am}$ in the solutions on the $\mathrm{U}$ and $\mathrm{Pu}$ values and that these minor elements are generally only present at $\leq 3 \%$ of the total actinide content, we can reasonably assume that regardless of whether or not the MEKED analysis includes the minor actinides, they will have no significant impact on the reported values for $\mathrm{U}$ and $\mathrm{Pu}$.

The attenuation matrix approach attempts to correct for the reduction in count rate across the KEdge transition of one element by the presence of the other elements relative to the reference spectrum. The general description of the method is somewhat counterintuitive and arises simply from the historical use of the concept of delta-mu (the change in attenuation across the K-Edge) and the use of a reference spectrum.

\section{$\underline{\text { Minor actinide performance }}$}

For our evaluation, only a few spectra collected with significant minor actinides present were available for evaluation of the MEKED algorithms. However, it appears that for low concentrations of minor actinides (approximately $<1 \mathrm{~g} / \mathrm{L}$ ) the minor actinide concentration 
reported was effectively the declaration value. For example, the spectra from a sample containing $\mathrm{U}, \mathrm{Pu}$, and $\mathrm{Np}$ (no Am present) was analyzed three times, and the only difference was the declared $\mathrm{Np}$ concentration. Each assay result essentially reported the declared Np concentration. The value of the $\mathrm{Np}$ declaration also appears to have a slight influence on the reported $\mathrm{U}$ concentration value.

We conclude that the MEKED analysis is not reporting a measured value for the minor actinide $\mathrm{Np}$, but rather the reported value is simply based on the ratio of the $\mathrm{U}$ and $\mathrm{Np}$ declarations scaled to the reported $\mathrm{U}$ result. A similar pattern was observed if Am was selected as the minor element. Unfortunately, we could find no discussion of the treatment of the minor elements in the CHKED software manual (Canberra document number 7067686 V1.0) or internal software help files.

Additional discussion on the minor actinides' performance is provided in Section 4.5.

Table 1. Impact of the minor actinide declaration value on the assay MEKED assay result (the same spectra were analyzed using different declarations).

\begin{tabular}{|l|l|l||l|l||l|l|}
\hline & $\begin{array}{l}\text { Declared } \\
(\mathrm{g} / \mathrm{L})\end{array}$ & $\begin{array}{l}\text { Reported } \\
\text { Concentration }(\mathrm{g} / \mathrm{L})\end{array}$ & $\begin{array}{l}\text { Declared } \\
(\mathrm{g} / \mathrm{L})\end{array}$ & $\begin{array}{l}\text { Reported } \\
\text { Concentration }(\mathrm{g} / \mathrm{L})\end{array}$ & $\begin{array}{l}\text { Declared } \\
(\mathrm{g} / \mathrm{L})\end{array}$ & $\begin{array}{l}\text { Reported } \\
\text { Concentration } \\
(\mathrm{g} / \mathrm{L})\end{array}$ \\
\hline $\mathrm{U}$ & 100.00 & $98.35 \pm 0.86 \%$ & 100.00 & $98.31 \pm 0.86 \%$ & 100.00 & $98.20 \pm 0.86 \%$ \\
\hline $\mathrm{Pu}$ & 7.00 & $7.38 \pm 12.64 \%$ & 7.00 & $7.24 \pm 12.88 \%$ & 7.00 & $7.21 \pm 12.94 \%$ \\
\hline $\mathrm{Np}$ & 4.00 & $3.94 \pm 11.39 \%$ & 1.00 & $0.99 \pm 11.39 \%$ & 0.30 & $0.30 \pm 20.72 \%$ \\
\hline
\end{tabular}

3. Why not include a matrix (solution) term in the attenuation matrix?

Since the attenuation matrix is a generalized approach, and for most HKED sample assays the sample density and concentrations of each actinide are included in the declaration, it may be beneficial to add another set of solvent attenuation parameters to the attenuation matrix. The solvent (or sample matrix) concentration varies with the actinide concentrations. Although the energy dependence of the sample matrix attenuation parameters is much slower than for the actinides, the sample matrix impacts the shape of the transmitted X-ray spectrum. The potential benefit of including a correction for the solvent concentration should be examined.

\section{Extrapolated KED analysis and the Attenuation Matrix}

The energy dependence of the interrogating X-ray spectrum requires that the transmission measurement be made through comparison to a reference spectrum (i.e., a nitric acid solution containing no actinides). The MEKED analysis defines two ROIs for each element, one above and one below the K-Edge transition energy (adjacent K-Edge transitions share the ROI defined between them). Each ROI from the sample spectrum is compared to the corresponding ROI in the reference spectrum. The concentration is determined by comparison of the transmission rates in the ROIs just above and below the K-Edge transition energy. This is referred to as the "non-extrapolated approach" in the traditional KED analysis. The presence of multiple actinides within the solution increases the complexity of the energy dependence of the sample's spectrum. The MEKED analysis addresses this complexity by introduction of the attenuation matrix.

However, implementation of the extrapolated analysis for the multi-elemental analysis, should not require the attenuation matrix. This is because each of the off-diagonal terms in the attenuation matrix tends to zero as the energy difference between the upper and lower ROIs approaches zero. 


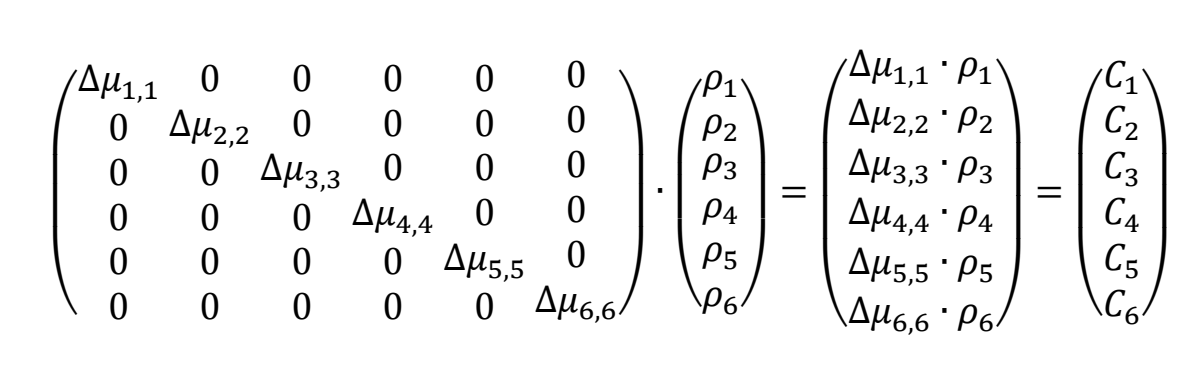

So that simply

$$
\rho_{z}=\frac{C_{z}}{\Delta \mu_{z, z}} .
$$

The traditional KED analysis provides for both an extrapolated and non-extrapolated analysis but did not implement an attenuation matrix. If our understanding of the matrix approach is correct, the MEKED extrapolated analysis should be identical to that of the traditional KED extrapolated analysis, at least for solutions containing no more than 3 actinides. If so, then the added value of the extrapolated MEKED approach is only to add additional elements to the analysis.

\section{Regions of Interest}

The small separation in energy between the K-Edge transitions of the actinides requires relatively narrow energy windows for the ROIs. Neglecting any Lorentzian broadening effects, the full width at half maximum (FWHM) of the observed K-Edge is approximately $550 \mathrm{eV}$, and the gap between left and right ROIs about a K-Edge transition is less than $1 \mathrm{keV}$ for most of the ROI windows. This separation is equivalent to only about 1.5 FWHM and ensures that the broadened edge will encroach into the ROI so that the observed count rates will be impacted by changes in the FWHM and any spectral drifting. More importantly, it will complicate the calculation of the attenuation matrix parameters.

Using the CHKED MEKED default settings, most of the ROIs are only 16 or 17 channels wide. For comparison, the traditional KED ROI widths are 23 or 24 channels wide. The reduced ROI widths result in a degradation of measurement precision by approximately $20 \%$ compared to the traditional analysis (e.g., $0.36 \%$ versus $0.3 \%$ for a single 1,000 s KED run).

Ideally, the separation from the K-Edge transition to the nearest ROI should be at least $2 \times$ FWHM for the typical KED HPGE detector (as was used with the traditional KED analysis) with $3 \times$ FWHM desired. Unfortunately, this rule of thumb would result in widths for the ROIs of less than $1 \mathrm{keV}$. Use of the more proper settings $(2 \times \mathrm{FWHM})$ will mitigate drift and resolution broadening effects but results in only 9 or 10 channels in width for the ROI and additional degradation in the measurement precision by

\begin{tabular}{|c|c|c|c|c|c|}
\hline \multicolumn{6}{|c|}{ Table 2. K-Edge transition energies and ROI windows for $\mathrm{U}$ and $\mathrm{Pu}$} \\
\hline \multicolumn{6}{|c|}{ CHKED MEKED K-Edge Default ROI limits for multi-elemental KED analysis } \\
\hline & K-Edge Energy & Left ROI Limits & Width (keV) & Right ROI Limits & Width (keV) \\
\hline $\mathrm{U}$ & 115.606 & $110.379 \quad 114.879$ & 4.5 & $116.388 \quad 117.888$ & 1.5 \\
\hline $\mathrm{Pu}$ & 121.797 & $119.484 \quad 120.984$ & 1.5 & $122.644 \quad 124.144$ & 1.5 \\
\hline \multicolumn{6}{|c|}{ Traditional KED ROI Limits } \\
\hline & K-Edge Energy & Left ROI Limits & Width (keV) & Right ROI Limits & Width $(\mathrm{keV})$ \\
\hline $\mathrm{U}$ & 115.606 & $107.200 \quad 113.300$ & 6.1 & 117.3 & 2.1 \\
\hline $\mathrm{Pu}$ & 121.797 & $117.300 \quad 119.400$ & 2.1 & 123.5 & 6.7 \\
\hline
\end{tabular}
approximately $30 \%$. 
The very narrow ROIs required for the MEKED limit the measurement precision, and in general prevent a full MEKED treatment for complex spectra with three or more major elemental constituents.

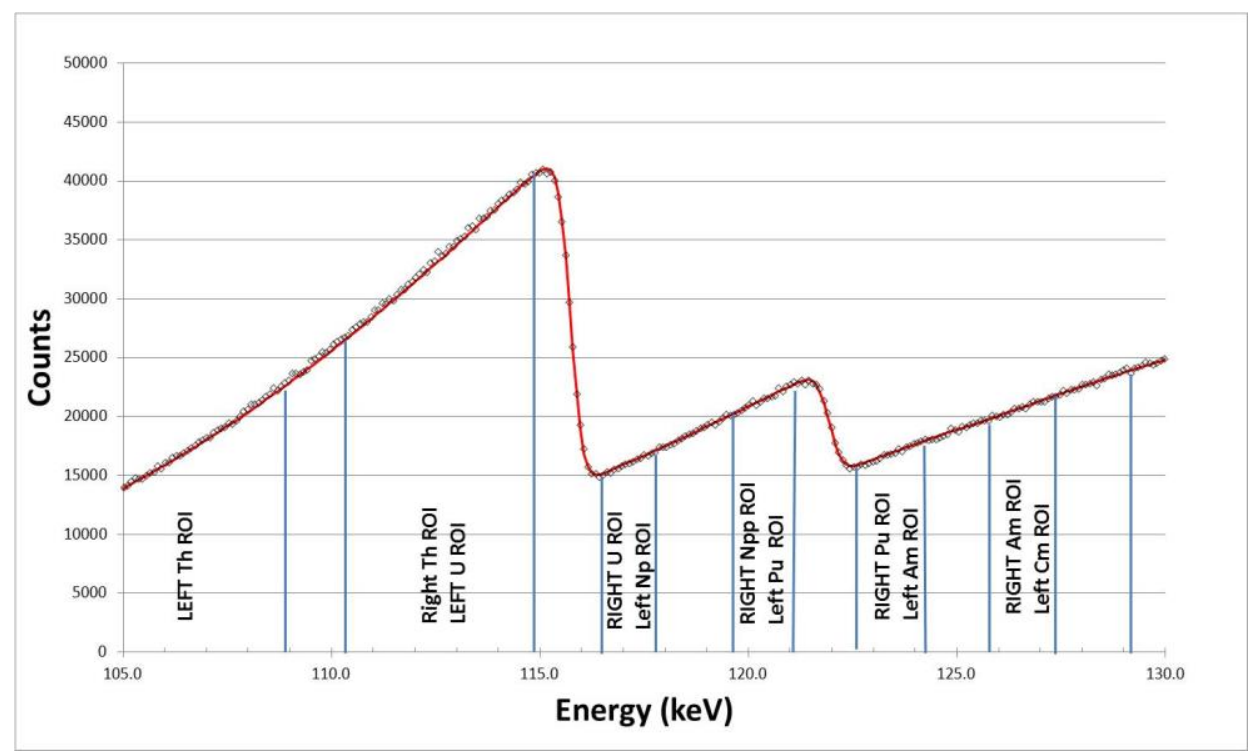

Figure 1. MEKED default ROIs overlain on a portion of the KED spectrum from a U, Pu sample. The ROIs tend to encroach into the high energy portion of the Gaussian-broadened K-Edge transitions.

\section{Other concerns}

We have identified significant deficiencies common to both the VMS HKED and CHKED analysis such as an improper background correction approach and the use of incorrect temperature correction coefficients. Both deficiencies result in biases significant in comparison to the reported concentration uncertainties. These effects and other less well understood interferences are discussed in this section.

1. Continuum (step background) correction

NOTE: A detailed discussion of this issue is provided in Appendix A.

The CHKED analysis includes the step background correction described in Ref. [1]. However, the ROIs used to determine the step background correction include significant fraction of counts that are not representative of the background below the K-Edge transitions. The result is an oversubtraction of the background, leading to biases in the reported actinide concentrations. The empirical calibrations used for the CHKED software effectively absorb these biases for assays performed near in time to the calibration. However, biases will begin to manifest as the ${ }^{109} \mathrm{Cd}$ source decays or the interrogating beam intensity is changed.

2. Temperature correction

The sample's temperature and, to a lesser extent, the temperature of the assay system will have an impact on the KED assay result. As the temperature changes, the density of the solution changes as well as the diameter of the sample vial. We note that the $0.05 \% /{ }^{\circ} \mathrm{C}$ deviation determined by Ottmar [1] is applicable to the rectangular glass cuvettes and not the polyethylene vials used with 
the ORNL HKED system. The observed change in assay result with temperature is $+0.11 \% /{ }^{\circ} \mathrm{C}$ for the cylindrical polyethylene (SGN) vial using the ORNL HKED system with the default correction applied (that is the correction appears to be applied in the wrong direction). Since the correction factor is not an adjustable parameter in the CHKED software, the temperature change will result in a positive systematic bias. Additional discussion about the temperature correction is provided in Appendix B.

3. Reference spectrum

Although the KED transmission measurements rely on count rate differences across the K-Edge transition to provide sensitivity and accuracy, the traditional and MEKED analyses determine the magnitude of the attenuation relative to a reference blank. However,

- The energy distribution from the X-ray generator changes as the X-ray tube ages. This effect will be small and have an impact similar to gain shift.

- The detected spectrum is influenced by electronic effects such as drift, dead-time and pile-up effects.

- Pile-up effects vary both with count rate and energy distribution.

Consider two spectra with the same overall count rate - one from W X-rays and one from ${ }^{109} \mathrm{Cd}$. The pile-up events from the $\mathrm{W}$ X-rays spectrum will be detected primarily in the range of $100-140 \mathrm{keV}$ photons while for pile-up events for ${ }^{109} \mathrm{Cd}$ will be detected as $176 \mathrm{keV}$ photons. For the KED analysis, the pile-up events from W X-rays directly interfere with the KED analysis, but those from ${ }^{109} \mathrm{Cd}$ would not. The KED spectra consist of a few peaks such as the W X-rays but is dominated by the transmitted Bremsstrahlung X-rays. So the pile-up effect from a reference blank will have a very different shape than one obtained from $300 \mathrm{~g}$ U/L even for equal count rates. These differences increase with increasing complexity of the solution.

Use of the reference spectrum for analysis of simple samples (e.g., $\mathrm{U}$ only) provides a reliable result because the differences in energy distribution are effectively absorbed by the empirical calibration. As long as the reference blank closely matches the characteristics of the samples (e.g., dimensions, chemical composition, and density) and the ${ }^{109} \mathrm{Cd}$ source activity of the sample measurement is not significantly different from that at calibration, this approach will not result in any significant bias. However, use of the reference spectrum with complex actinide samples may introduce additional biases as the shape of the transmission spectrum becomes correspondingly complex.

\section{Sample vial size}

The sample vial thickness cannot be adjusted on a per assay basis without altering the counter configuration in the CHKED software. We note that this issue may have been already addressed by the vendor in a recent release of the software. 


\section{GENERAL COMMENTS ON THE CHKED SOFTWARE}

\section{SetuplCertificates and Declarations}

- Only allow definition of three elements.

- Occasionally, definitions could not be saved even though no errors were evident.

- Round off to two significant digits in uncertainty entries, but IDMS results may report small uncertainties to three decimal places (e.g., 0.115). Because of the high degree of precision of the CHKED measurements and the accuracy of the Isotope Dilution Mass Spectrometry (IDMS) results, three decimal places are recommended.

\section{SetuplSample Container Declaration}

- Consider adding an entry for container wall thickness

\section{Setup\Sample Type Processing Parameters}

- Only allows definition of three elements.

- Default ROIs for U, Pu, and Am are not the same as noted in KfK4590 or Ref. [3].

\section{Temperature Correction}

- Parameters cannot be entered from the setup screens - values used are those predefined from KfK4590.

- These parameters are not representative of current generation HKED systems using the SGN vials.

- The temperature correction parameters are not visible to the user.

\section{Data Review}

The data review program is intended to allow review and reanalysis of HKED files to reevaluate problematic assay results on an individual basis and to reanalyze multiple data sets with revised parameters. The data review tool provided with CHKED is a dramatic improvement over those available with the traditional VMS software. However, in general the data review application is difficult to navigate, and certain key parameters are not readily accessible to the user. For example, it was not possible to

- select a certificate file in data review,

- add a minor or major element to the declaration,

- add a second minor element, or

- add a minor element if a second major element had been defined.

A fairly common entry mistake is to select the wrong declaration or certificate. A drop-down menu to allow selection of alternate declarations or to create a new one would be beneficial. For example, if an 
unexpected element were to be found in the solution, currently the only way to include this in the reanalysis is to modify the original certificate and reanalyze using the review software's batch mode.

- MEKED only accommodates elements assigned to the sample vial in the counter definition menus. This again complicates the data review because the user must back completely out of data review and redefine the calibration.

- Help files do not define the Analysis Sequence File (ASF) steps available or the relevant report sections.

\section{Editing a Calibration}

The CHKED software provides an option to edit an existing calibration. We note that editing a calibration does not change the calibration date in data review, so it is not obvious that a calibration file has been revised.

\section{CHKED Analysis Tools}

The CHKED software provides a number of utilities to assist in calibration and data review.

\section{Calibration graphics}

Graphics have been added to aid in the evaluation of the calibration process. For example, the KED calibration curve is shown in Figure 2 below. However, because of the range in concentrations and the relatively small errors associated with the KED assay, it is difficult to see significant deviations from the fitted line. A more beneficial plot would be the relative difference as a function of concentration.

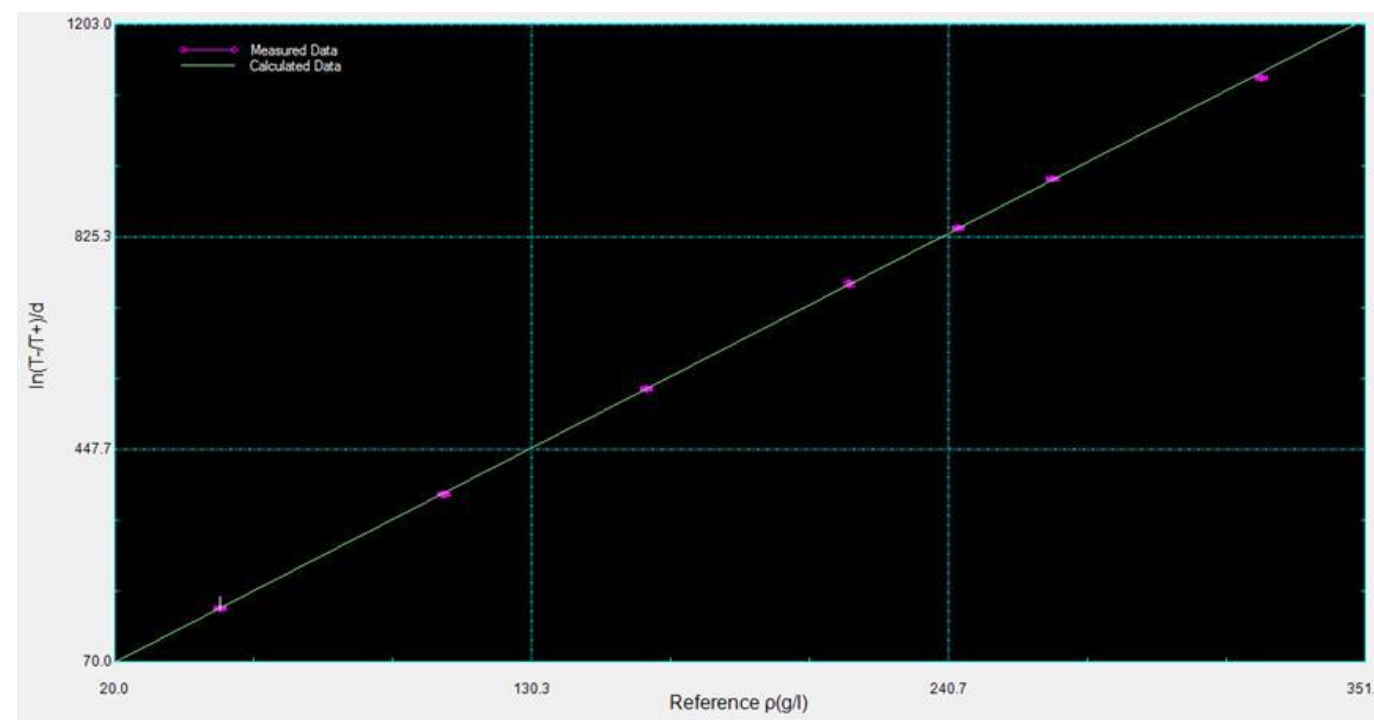

Figure 2. KED calibration curve for uranium-only solutions.

\section{Data Analysis using the Simulator}

The Genie $2 \mathrm{k}$ simulator tool can be used to import data files into the CHKED database. This tool was used extensively in our evaluation of the CHKED software, and although this is a useful tool, it is simple to enter erroneous or fictitious data into the system. 
- The simulated data acquisition spectra are not the same as the imported data files. Following completion (or interruption) of the simulated assay sequence, the new data file briefly appears and is then replaced by the next data acquisition sequence. Care must be taken to ensure that the proper files are being imported.

- It is possible to import the data files with a different sample container (allowing the sample size to be altered during reanalysis).

- It is possible to "acquire" the new data file with an incorrect X-ray tube setting. Although this does not appear to impact the "sample assays," it does impact the reference measurement. Since the sample spectrum is ratioed to the reference spectrum and adjusted for relative beam intensity, it is possible to inadvertently or deliberately affect the reported assay results.

Neither the reference manuals or help files accurately describe the necessary steps for use of the simulation tool for importing spectra.

\subsection{CHKED REPORTS}

As a general comment, the CHKED reporting templates follow fairly closely to the original VMS templates. However, both could use some improvement. For example, in the MEKED report template the reported ${ }^{109} \mathrm{Cd}$ reference peak rate is actually the total counts in the peak.

\section{MEKED Calibration Report}

The KED calibration report is similar to that of the VMS predecessor; however, both lack relevant information for evaluation of the calibration and contain some ambiguous entries (e.g., the measured concentration values refer to the assay results calculated with the previous calibration parameters, but this is not stated in the report). Nor do the certificate values (i.e., declared concentrations) appear in the calibration report. Because the CHKED software tags the raw data files (i.e., the .CNF files) with a sequence number and does not provide a descriptive file name, the calibration report should list the assay sequence number. Other useful information includes the HV and current settings for the X-ray generator, measurement cell temperature, and sample vial diameter. It is assumed that relevant corrections are applied to each file during the calibration processing, the report should indicate this.

It is suggested that the previous (now obsolete) assay results be removed from the first section of the report and replaced with the assay sequence number, HV, and current settings. The assay results using the "older" parameters should be moved to the end of the report and listed side by side with the updated values based on the new calibration parameters.

The tabulated delta-mu values in the 2 nd section of the report should also provide the calculated uncertainty for the value.

\section{PERFORMANCE OF THE CHKED K-EDGE TRANSMISSION ANALYSIS METHODS}

The CHKED software performance was evaluated for its four primary analysis methods, the three socalled "classic VMS methods" and the Canberra-devised MEKED. The VMS methods include the traditional KED, the stand-alone X-ray fluorescence (XRF), and Hybrid analyses. Our evaluation of these three methods indicate that the performance of their implementation within the Windows-based CHKED software is unchanged from the earlier VMS-based HKED software with the possible exception of the KED temperature correction. The MEKED analysis affords some additional capabilities for the analysis of complex actinide mixtures but (at least the version available to us (V 1.0) cannot be considered 
finished or robust. (For example, the MEKED analysis treats only two elements in a "primary" fashion, additional impurity elements are treated as interferences, and the assay result for the third element is frequently statistically indistinguishable from the entry in the sample declaration even if it is many times greater than the true value.) However, the MEKED analysis does offer important advantages over the traditional KED analysis method, in that the reported result for the second primary actinide is generally accurate. The remainder of this section will discuss the measurement performance observed using ORNL standards and spectra provided from the On-site Laboratory at the Rokkasho Reprocessing Plant.

\subsection{KED TRANSMISSION ANALYSIS}

The traditional KED transition analysis is performed when a single actinide (typically uranium) is present with relatively small amounts (e.g., U:Pu 100) of additional actinides present. The measurement performance of the traditional KED analysis was examined first by repeated measurements of a number of uranium-only solution standards at ORNL. The temperature-corrected assay results for these runs are provided in Table 3. The table provides the expected measurement precision as determined in Ref. [1], the measurement precision reported on the CHKED printouts, and the measurement precision that would be obtained with a more accurate temperature correction. We can see that the observed measurement precision from the CHKED printouts is slightly better than the expected values. Finally, with the proper temperature correction applied, additional improvement in measurement precision is expected.

\begin{tabular}{|c|c|c|c|c|}
\hline \multicolumn{5}{|c|}{$\begin{array}{c}\text { Table 3. Typical measurement precision for the CHKED KED assay for } \\
\text { uranium solutions in the SGN vial (1,000 s assay times). The precision } \\
\text { obtained after correcting the temperature correction factor. }\end{array}$} \\
\hline$[\mathrm{U}] \mathrm{g} / \mathrm{L}$ & $\begin{array}{c}\# 1,000 \mathrm{~s} \\
\text { runs }\end{array}$ & $\begin{array}{c}\text { Expected } \\
\text { Precision [1] }\end{array}$ & $\begin{array}{c}\text { CHKED } \\
\text { Precision }\end{array}$ & $\begin{array}{c}\text { Corrected CHKED } \\
\text { Precision }\end{array}$ \\
\hline 48.27 & 9 & $0.70 \%$ & $0.77 \%$ & $0.67 \%$ \\
107.3 & 15 & $0.39 \%$ & $0.33 \%$ & $0.31 \%$ \\
214.61 & 9 & $0.28 \%$ & $0.32 \%$ & $0.28 \%$ \\
268.21 & 18 & $0.28 \%$ & $0.22 \%$ & $0.18 \%$ \\
321.91 & 15 & $0.30 \%$ & $0.28 \%$ & $0.22 \%$ \\
\hline
\end{tabular}

The measurement biases were examined by comparison of the reported assay results with declared values for several uranium and plutonium solution working standards. (Assay times for these measurements varied from 1 hour to several hours.) Measurements results from the ORNL HKED system are provided in Table 4. The observed deviations between measured and declared values are consistent with the systematic uncertainty in the calibration standards and measurement precision as shown in Figure 3. From these results we conclude that the CHKED software does not introduce additional uncertainty during the analysis of a simple actinide solution (e.g., $\mathrm{U}$ only) in comparison to historically observed performance of the KED measurement. 


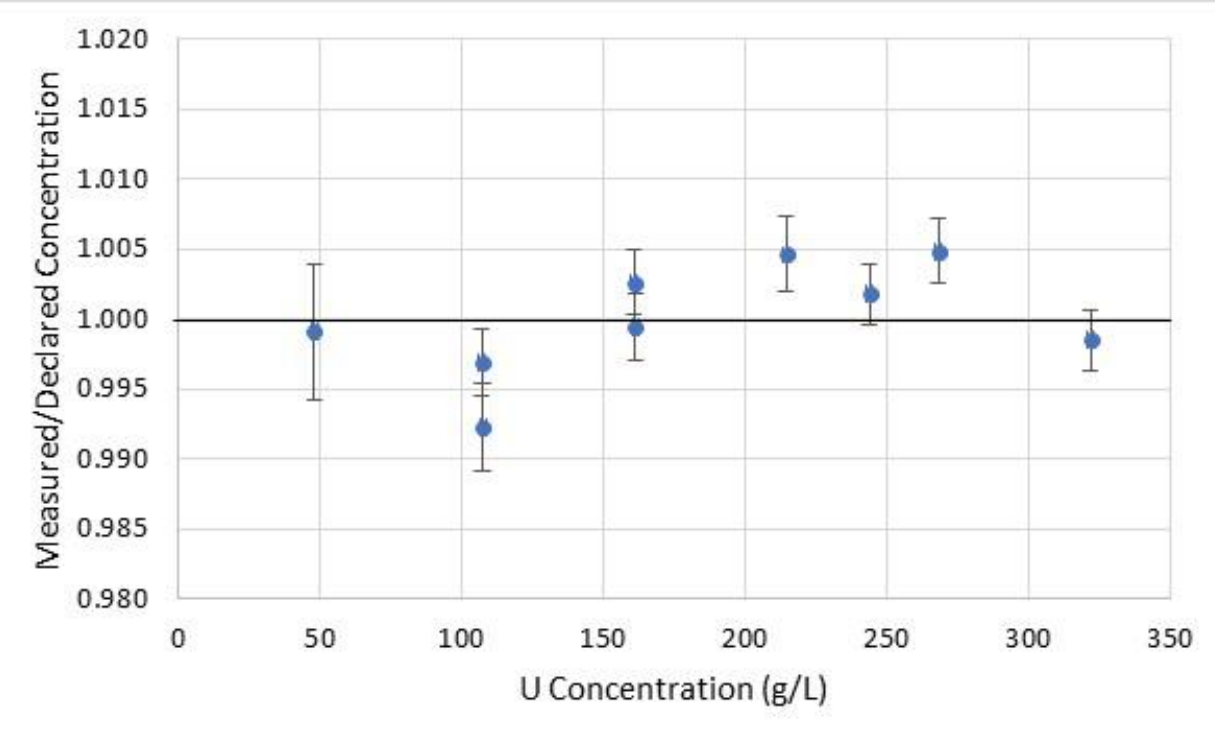

Figure 3. Plot of the ratio of measured-to-declared uranium concentration values for the ORNL calibration standards.

\begin{tabular}{|l|cc|cc|cc|}
\hline \multicolumn{2}{|c|}{ Table 4. Typical measurement biases observed for two different HKED systems. } \\
\hline Standard & \multicolumn{2}{|c|}{ Declared } & \multicolumn{2}{|c|}{ Measured Concentration } \\
Concentration (g U/L)
\end{tabular}

\subsection{PERFORMANCE WITH PLUTONIUM AS THE PRIMARY ELEMENT}

High-concentration plutonium solutions were not available at ORNL for this evaluation; consequently, we have examined the Pu solution spectra provided by the IAEA for this study. These were used to examine measurement precision and potential biases for plutonium-only assay using the CHKED software. Typical IDMS uncertainties of $\pm 0.1 \%$ were assumed for the declared concentrations. Analysis of the data provided indicates that assay results provided by the CHKED software are consistent with previous results using the VMS-based software. Where plutonium is the dominant actinide, the measurement precision is roughly 50\% larger than observed for the uranium-only runs. For a single dominant actinide, the CHKED MEKED measurement performance is equivalent to that obtained using the traditional KED transmission analysis. 


\begin{tabular}{|c|c|c|c|c|c|}
\hline \multirow{2}{*}{$\begin{array}{c}\text { Declared } \\
\text { Value } \\
{[\mathrm{Pu}](\mathrm{g} / \mathrm{L})}\end{array}$} & \multirow{2}{*}{$\begin{array}{c}\text { Assay } \\
\text { Result } \\
{[\mathrm{Pu}](\mathrm{g} / \mathrm{L})} \\
\end{array}$} & \multicolumn{2}{|c|}{ Absolute Uncertainty } & \multicolumn{2}{|c|}{ Relative Uncertainty } \\
\hline & & Precision & Uncertainty & Precision & Uncertainty \\
\hline 56.5 & 56.28 & \pm 0.51 & \pm 0.53 & $\pm 0.9 \%$ & $\pm 0.9 \%$ \\
\hline 152.74 & 151.71 & \pm 0.41 & \pm 0.56 & $\pm 0.3 \%$ & $\pm 0.4 \%$ \\
\hline 169.41 & 166.06 & \pm 0.40 & \pm 0.58 & $\pm 0.2 \%$ & $\pm 0.4 \%$ \\
\hline
\end{tabular}

\subsection{HYBRID ANALYSIS}

The HKED Hybrid analysis uses the KED transmission measurement to provide the uranium concentration for the sample. The XRF measurement determines the relative elemental concentrations (i.e., U:Pu and U:Am). The Hybrid analysis combines these two results to provide concentration values for $\mathrm{U}, \mathrm{Pu}$, and $\mathrm{Am}$. The performance of the CHKED software operated in the Hybrid mode was examined using two types of samples. The first are typical of the 100:1 U:Pu solutions that the HKED originally targeted. The second group consists of a series of higher plutonium concentrations with U:Pu ratios in the range of $1-2$.

Table 6 shows the measurement results for the more traditional solutions where the plutonium content is low compared to the uranium concentration. For these items, there is good agreement between the measured and declared values. The CHKED performance in this mode is consistent with the historical VMS-based analysis. For the ORNL system, the typical uncertainty observed for the U:Pu ratio is $\sim 1 \%$ for these materials.

\begin{tabular}{|c|c|c|c|cc|cc|rr|}
\hline \multicolumn{9}{|c|}{ Table 6. CHKED Hybrid mode analysis results for U:Pu >10. } \\
\hline \multirow{2}{*}{ Item } & \multicolumn{2}{|c|}{ Declared Values } & \multicolumn{2}{|c|}{ CHKED Hybrid Analysis Results } \\
\cline { 2 - 11 } & {$[\mathrm{U}] \mathrm{g} / \mathrm{L}$} & {$[\mathrm{Pu}] \mathrm{g} / \mathrm{L}$} & $\mathrm{U}: P u$ Ratio & \multicolumn{2}{|c|}{$[\mathrm{U}] \mathrm{g} / \mathrm{L}$} & \multicolumn{2}{|c|}{$[\mathrm{Pu}] \mathrm{g} / \mathrm{L}$} & \multicolumn{2}{c|}{$\mathrm{U}: \mathrm{Pu}$ Ratio } \\
\hline UPU100 & 107.65 & 1.0351 & 104.00 & 106.81 & \pm 0.26 & 1.040 & \pm 0.009 & 102.7 & \pm 0.8 \\
UPU150 & 161.42 & 1.5573 & 103.65 & 161.33 & \pm 0.19 & 1.573 & \pm 0.011 & 102.6 & \pm 0.7 \\
UPU250 & 244.04 & 2.9152 & 83.71 & 244.48 & \pm 0.20 & 2.942 & \pm 0.017 & 83.1 & \pm 0.5 \\
\hline UM-1 & 100.60 & 7.81 & 12.88 & 101.08 & \pm 0.32 & 7.736 & \pm 0.28 & 13.05 & \pm 0.03 \\
\hline
\end{tabular}

As the relative plutonium concentration increases, some degradation is expected in the performance of the Hybrid mode analysis. Biases are introduced by increased fluorescence of uranium X-rays by plutonium $\mathrm{X}$-rays, preferential fluorescence of the higher-Z actinides, and increased interference between X-rays as otherwise negligible X-ray peaks begin to build in the key ROIs in the spectrum. The MOX data sets provided were analyzed using calibrations developed for the traditional U:Pu ratios of 100:1, and the results are presented in Table 7. Table 8 provides a summary of the biases for these measurement results. The reported uranium concentrations produced only a slight average bias (consistent with typical IDMS measurement uncertainty).

- The measurement precision is approximately two times larger than the uranium-only measurement primarily due to the attenuation of the interrogating flux by the plutonium. This observed performance generally agrees with expectation for the stated concentration ranges. 
- The plutonium results exhibit a significant negative bias $(-3.4 \%)$, which could in principle be improved by calibration with representative materials. However, the observed random error component (1.9\%) exceeds the expectations of the International Target Values (ITV) [4] and is not readily correctable with the current analysis methodology. Items

\begin{tabular}{|c|c|c|c|c|c|c|c|c|c|c|c|}
\hline \multirow{3}{*}{$\frac{\text { Item }}{1}$} & \multicolumn{3}{|c|}{ Declared Values } & \multicolumn{8}{|c|}{ CHKED Hybrid Analysis Results } \\
\hline & \multirow{2}{*}{$\frac{[\mathrm{U}] \mathrm{g} / \mathrm{L}}{178.37}$} & \multirow{2}{*}{$\frac{[\mathrm{Pu}] \mathrm{g} / \mathrm{L}}{82.67}$} & \multirow{2}{*}{$\frac{\mathrm{U}: \mathrm{Pu} \text { Ratio }}{2.16}$} & \multicolumn{2}{|c|}{$[\mathrm{U}] \mathrm{g} / \mathrm{L}$} & \multicolumn{2}{|c|}{ U:Pu Ratio } & \multicolumn{2}{|c|}{$[\mathrm{Pu}] \mathrm{g} / \mathrm{L}$} & \multicolumn{2}{|c|}{$[\mathrm{Am}] \mathrm{g} / \mathrm{L}$} \\
\hline & & & & 178.44 & \pm 0.80 & 2.250 & \pm 0.003 & 78.99 & \pm 0.26 & 1.72 & \pm 0.03 \\
\hline 3 & 229.07 & 105.99 & 2.16 & 226.67 & \pm 1.04 & 2.280 & \pm 0.003 & 99.32 & \pm 0.32 & 2.15 & \pm 0.04 \\
\hline 4 & 229.07 & 105.99 & 2.16 & 231.23 & \pm 0.69 & 2.280 & \pm 0.003 & 101.28 & \pm 0.28 & 2.21 & \pm 0.04 \\
\hline 5 & 228.74 & 105.80 & 2.16 & 227.48 & \pm 1.11 & 2.270 & \pm 0.003 & 100.08 & \pm 0.32 & 2.15 & \pm 0.04 \\
\hline 6 & 228.74 & 105.80 & 2.16 & 230.02 & \pm 0.74 & 2.280 & \pm 0.003 & 101.10 & \pm 0.29 & 2.22 & \pm 0.04 \\
\hline 7 & 118.46 & 99.40 & 1.19 & 118.55 & \pm 0.78 & 1.200 & \pm 0.001 & 97.54 & \pm 0.40 & 2.34 & \pm 0.03 \\
\hline 8 & 225.33 & 103.59 & 2.18 & 226.61 & \pm 0.88 & 2.220 & \pm 0.003 & 101.52 & \pm 0.31 & 2.58 & \pm 0.04 \\
\hline 9 & 225.33 & 103.59 & 2.18 & 226.49 & \pm 0.91 & 2.230 & \pm 0.003 & 101.58 & \pm 0.33 & 2.69 & \pm 0.04 \\
\hline 10 & 225.33 & 103.59 & 2.18 & 226.76 & \pm 0.73 & 2.230 & \pm 0.003 & 101.34 & \pm 0.30 & 2.71 & \pm 0.04 \\
\hline 11 & 171.18 & 78.71 & 2.17 & 171.87 & \pm 0.67 & 2.190 & \pm 0.003 & 78.13 & \pm 0.25 & 1.89 & \pm 0.03 \\
\hline
\end{tabular}

\begin{tabular}{|c|cc|cc|cc|}
\hline \multicolumn{8}{|c|}{ Table 8. Assay bias resulting from the CHKED Hybrid K-Edge analysis of } \\
the MOX spectra provided for this study. \\
\hline Item & \multicolumn{2}{|c|}{$[\mathrm{U}]$ assay Bias } & \multicolumn{2}{|c|}{$[\mathrm{Pu}]$ Assay Bias } & \multicolumn{2}{c|}{ U:Pu Bias } \\
\hline 1 & $0.04 \%$ & $\pm 0.45 \%$ & $-4.5 \%$ & $\pm 0.32 \%$ & $4.1 \%$ & $\pm 0.14 \%$ \\
3 & $-1.05 \%$ & $\pm 0.46 \%$ & $-6.3 \%$ & $\pm 0.30 \%$ & $5.5 \%$ & $\pm 0.13 \%$ \\
4 & $0.94 \%$ & $\pm 0.30 \%$ & $-4.4 \%$ & $\pm 0.27 \%$ & $5.5 \%$ & $\pm 0.13 \%$ \\
5 & $-0.55 \%$ & $\pm 0.49 \%$ & $-5.4 \%$ & $\pm 0.30 \%$ & $5.0 \%$ & $\pm 0.13 \%$ \\
6 & $0.56 \%$ & $\pm 0.32 \%$ & $-4.4 \%$ & $\pm 0.28 \%$ & $5.5 \%$ & $\pm 0.13 \%$ \\
7 & $0.07 \%$ & $\pm 0.66 \%$ & $-1.9 \%$ & $\pm 0.40 \%$ & $0.7 \%$ & $\pm 0.12 \%$ \\
8 & $0.57 \%$ & $\pm 0.39 \%$ & $-2.0 \%$ & $\pm 0.30 \%$ & $2.1 \%$ & $\pm 0.12 \%$ \\
9 & $0.51 \%$ & $\pm 0.40 \%$ & $-1.9 \%$ & $\pm 0.31 \%$ & $2.5 \%$ & $\pm 0.12 \%$ \\
10 & $0.63 \%$ & $\pm 0.32 \%$ & $-2.2 \%$ & $\pm 0.29 \%$ & $2.5 \%$ & $\pm 0.12 \%$ \\
11 & $0.40 \%$ & $\pm 0.39 \%$ & $-0.7 \%$ & $\pm 0.32 \%$ & $0.7 \%$ & $\pm 0.13 \%$ \\
\hline Average & \multicolumn{2}{|c|}{$0.21 \%$} & \multicolumn{2}{|c|}{$-3.37 \%$} & \multicolumn{2}{c|}{$3.40 \%$} \\
\hline St. Dev. & \multicolumn{2}{|c|}{$0.61 \%$} & \multicolumn{2}{|c|}{$1.85 \%$} & \multicolumn{1}{c|}{$1.94 \%$} \\
\hline
\end{tabular}

The calibration applied to the data in Table 7 was developed for $\mathrm{U}: \mathrm{Pu}$ concentrations ratios of $\sim 100: 1$. To provide a more realistic assessment of the performance, the results of the measurements were adjusted to account for the average bias (e.g., a recalibration was performed). The bias reported in the Table 9 are more representative of a properly calibrated HKED Hybrid analysis where the plutonium cannot be considered an impurity. The revised results indicate that with proper calibration the average bias reported for the Hybrid analysis will be small; however, the random errors associated with the Pu analysis will be 
on the order of $2 \%$. This level of performance for the Hybrid mode is somewhat better than expected, but it illustrates the need for implementation of a true multi-elemental analysis.

\begin{tabular}{|c|cc|cc|cc|}
\hline \multicolumn{6}{|c|}{$\begin{array}{c}\text { Table 9. Assay bias resulting from the CHKED Hybrid K-Edge analysis } \\
\text { of spectra provided from a reprocessing facility after recalibration. }\end{array}$} \\
\hline Item & \multicolumn{2}{|c|}{$[\mathrm{U}]$ assay Bias } & \multicolumn{2}{|c|}{$[\mathrm{Pu}]$ Assay Bias } & \multicolumn{2}{c|}{ U:Pu Bias } \\
\hline 1 & $0.04 \%$ & $\pm 0.45 \%$ & $-0.8 \%$ & $\pm 0.33 \%$ & $0.7 \%$ & $\pm 0.13 \%$ \\
3 & $-1.05 \%$ & $\pm 0.46 \%$ & $-3.0 \%$ & $\pm 0.31 \%$ & $2.1 \%$ & $\pm 0.12 \%$ \\
4 & $0.94 \%$ & $\pm 0.30 \%$ & $-1.1 \%$ & $\pm 0.28 \%$ & $2.1 \%$ & $\pm 0.12 \%$ \\
5 & $-0.55 \%$ & $\pm 0.49 \%$ & $-2.1 \%$ & $\pm 0.31 \%$ & $1.6 \%$ & $\pm 0.12 \%$ \\
6 & $0.56 \%$ & $\pm 0.32 \%$ & $-1.4 \%$ & $\pm 0.29 \%$ & $2.0 \%$ & $\pm 0.12 \%$ \\
7 & $0.07 \%$ & $\pm 0.66 \%$ & $2.7 \%$ & $\pm 0.42 \%$ & $-2.6 \%$ & $\pm 0.12 \%$ \\
8 & $0.57 \%$ & $\pm 0.39 \%$ & $1.9 \%$ & $\pm 0.31 \%$ & $-1.3 \%$ & $\pm 0.12 \%$ \\
9 & $0.51 \%$ & $\pm 0.40 \%$ & $1.4 \%$ & $\pm 0.32 \%$ & $-0.8 \%$ & $\pm 0.12 \%$ \\
10 & $0.63 \%$ & $\pm 0.32 \%$ & $1.5 \%$ & $\pm 0.30 \%$ & $-0.8 \%$ & $\pm 0.12 \%$ \\
11 & $0.40 \%$ & $\pm 0.39 \%$ & $3.1 \%$ & $\pm 0.33 \%$ & $-2.6 \%$ & $\pm 0.13 \%$ \\
\hline Average & \multicolumn{2}{|c|}{$0.21 \%$} & \multicolumn{2}{|c|}{$0.20 \%$} & \multicolumn{2}{c|}{$0.03 \%$} \\
\hline St. Dev. & $0.61 \%$ & \multicolumn{2}{|c|}{$2.15 \%$} & \multicolumn{3}{c}{$1.88 \%$} \\
\hline
\end{tabular}

\subsection{PERFORMANCE OF THE CHKED MEKED ANALYSIS}

The CHKED MEKED analysis, discussed in Section 2, attempts to provide a multi-elemental analysis for the more complex actinide mixtures using only the KED transmission spectrum. The KED spectra from the samples examined in Section 4.3 were analyzed using the MEKED analysis, and the results are reported in Table 10. The average bias for the reported $\mathrm{U}$ and $\mathrm{Pu}$ concentrations for the collection of MOX samples is relatively small and is consistent with typical calibration uncertainties for the traditional KED analysis.

\begin{tabular}{|c|c|c|c|c|c|c|c|c|c|c|}
\hline \multicolumn{3}{|c|}{ Declared Values } & \multicolumn{8}{|c|}{ CHKED MEKED Analysis Results } \\
\hline$[\mathrm{U}] \mathrm{g} / \mathrm{L}$ & {$[\mathrm{Pu}] \mathrm{g} / \mathrm{L}$} & U:Pu Ratio & \multicolumn{2}{|c|}{$[\mathrm{U}](\mathrm{g} / \mathrm{L})$} & \multicolumn{2}{|c|}{$[\mathrm{Pu}](\mathrm{g} / \mathrm{L})$} & \multicolumn{2}{|c|}{$[\mathrm{Am}](\mathrm{g} / \mathrm{L})$} & \multicolumn{2}{|c|}{ U:Pu Ratio } \\
\hline 229.07 & 105.99 & 2.16 & 226.28 & \pm 0.27 & 106.31 & \pm 0.27 & 2.23 & \pm 0.01 & 2.129 & \pm 0.006 \\
\hline 229.07 & 105.99 & 2.16 & 230.21 & \pm 0.44 & 107.00 & \pm 0.96 & 1.17 & \pm 0.04 & 2.152 & \pm 0.020 \\
\hline 228.74 & 105.80 & 2.16 & 227.39 & \pm 0.60 & 104.91 & \pm 0.71 & 0.42 & \pm 0.03 & 2.167 & \pm 0.016 \\
\hline 228.74 & 105.80 & 2.16 & 229.72 & \pm 0.11 & 106.81 & \pm 0.18 & 0.22 & \pm 0.01 & 2.151 & \pm 0.004 \\
\hline 118.46 & 99.40 & 1.19 & 117.48 & \pm 0.52 & 99.67 & \pm 0.39 & 5.57 & \pm 0.02 & 1.179 & \pm 0.007 \\
\hline 225.33 & 103.59 & 2.18 & 225.58 & \pm 0.65 & 104.75 & \pm 0.83 & 2.17 & \pm 0.03 & 2.153 & \pm 0.018 \\
\hline 225.33 & 103.59 & 2.18 & 226.69 & \pm 0.14 & 104.04 & \pm 0.78 & 2.12 & \pm 0.03 & 2.179 & \pm 0.016 \\
\hline 225.33 & 103.59 & 2.18 & 225.51 & \pm 0.73 & 103.99 & \pm 0.51 & 2.13 & \pm 0.02 & 2.169 & \pm 0.013 \\
\hline 171.18 & 78.71 & 2.17 & 171.48 & \pm 0.25 & 78.29 & \pm 0.08 & 0.98 & \pm 0.01 & 2.190 & \pm 0.004 \\
\hline
\end{tabular}


The average 1,000 second measurement precision for these runs is $0.36 \%$ for uranium and $0.89 \%$ for plutonium. For a typical $3 \times 1,000 \mathrm{~s}$ assay sequence, the measurement precision for uranium is $0.21 \%$ and for plutonium it is $0.50 \%$ when all three spectra pass internal quality control checks. Table 11 provides the relative bias and uncertainty for each of these samples. We see that although the measurement precision reported for each sample is sufficient to meet the International Target Values [4], the overall measurement performance does not. The random uncertainty component for uranium exceeds the target ( $0.2 \%$ random, $0.2 \%$ systematic, $0.28 \%$ combined), and for plutonium, the observed performance is nominal compared to the targets ( $0.8 \%$ random, $0.5 \%$ systematic, $0.94 \%$ combined).

\begin{tabular}{|c|c|c|c|c|c|c|}
\hline Item & \multicolumn{2}{|c|}{ [U] assay Bias } & \multicolumn{2}{|c|}{$[\mathrm{Pu}]$ Assay Bias } & \multicolumn{2}{|c|}{$\mathrm{U}: \mathrm{Pu}$ Bias } \\
\hline 1 & $(0.03$ & $\pm 0.46) \%$ & - & - & - & - \\
\hline 3 & $(-1.05$ & $\pm 0.46) \%$ & - & - & - & - \\
\hline 4 & $(-1.22$ & $\pm 0.15) \%$ & $(0.3$ & $\pm 0.27) \%$ & $(-1.44$ & $\pm 0.28) \%$ \\
\hline 5 & $(0.49$ & $\pm 0.22) \%$ & $(0.95$ & $\pm 0.91) \%$ & $(-0.38$ & $\pm 0.93) \%$ \\
\hline 6 & $(-0.6$ & $\pm 0.28) \%$ & $(-0.85$ & $\pm 0.68) \%$ & $(0.32$ & $\pm 0.74) \%$ \\
\hline 7 & $(0.42$ & $\pm 0.11) \%$ & $(0.95$ & $\pm 0.20) \%$ & $(-0.42$ & $\pm 0.19) \%$ \\
\hline 8 & $(-0.83$ & $\pm 0.45) \%$ & $(0.27$ & $\pm 0.40) \%$ & $(-0.93$ & $\pm 0.59) \%$ \\
\hline 9 & $(0.11$ & $\pm 0.31) \%$ & $(1.11$ & $\pm 0.81) \%$ & $(-1.24$ & $\pm 0.83) \%$ \\
\hline 10 & $(0.6$ & $\pm 0.12) \%$ & $(0.43$ & $\pm 0.76) \%$ & $(-0.05$ & $\pm 0.73) \%$ \\
\hline 11 & $(0.07$ & $\pm 0.34) \%$ & $(0.38$ & $\pm 0.50) \%$ & $(-0.51$ & $\pm 0.60) \%$ \\
\hline Average & \multicolumn{2}{|c|}{$-0.16 \%$} & \multicolumn{2}{|c|}{$0.34 \%$} & \multicolumn{2}{|c|}{$-0.41 \%$} \\
\hline St. Dev. & \multicolumn{2}{|c|}{$0.65 \%$} & \multicolumn{2}{|c|}{$0.66 \%$} & \multicolumn{2}{|c|}{$0.75 \%$} \\
\hline
\end{tabular}

Comparison of the U:Pu values determined via the Hybrid and MEKED Analysis

The measurement precision for the hybrid assay is two to three times better than achieved for the same samples with the MEKED analysis. However, the overall error in the reported U:Pu ratio is lower using the MEKED analysis $(0.75 \%)$ than is achieved using the Hybrid analysis (1.9\%).

\subsection{IMPACT OF THE AM DECLARATION ON THE ASSAY RESULT.}

We have noted a correlation between the declared Am concentration and the Am concentration reported by the MEKED analysis. However, the correlation is not consistent. Examples are shown in Table 12 for the two types of correlation observed. In the first type, the declared Am is roughly echoed back by the analysis. That is if the Am is declared at $5 \mathrm{~g} / \mathrm{L}$, the assay result produces a similar value. For the same assay (same CNF files), analysis with a declaration of $0.2 \mathrm{~g} \mathrm{Am} / \mathrm{L}$ returns a value of the same magnitude (e.g., $0.37 \mathrm{~g} / \mathrm{L}$ ). Other times the impact on the assay result is less dramatic, as shown in the second half of the table. In this case, the reported Am concentration changes only a few percent. In both cases the reported $\mathrm{U}$ and $\mathrm{Pu}$ concentrations are affected to a small extent. 


\begin{tabular}{|c|c|c|cc|cr|}
\hline \multicolumn{7}{|c|}{ Table 12. Impact of the Am declaration value of the non-extrapolated assay result for two } \\
samples. \\
\hline Declared $[\mathrm{Am}](\mathrm{g} / \mathrm{L})$ & \multicolumn{2}{|c|}{$[\mathrm{U}](\mathrm{g} / \mathrm{L})$} & \multicolumn{2}{|c|}{$[\mathrm{Pu}](\mathrm{g} / \mathrm{L})$} & \multicolumn{2}{l|}{$[\mathrm{Am}](\mathrm{g} / \mathrm{L})$} \\
\hline $0.2 \mathrm{~g} / \mathrm{L}$ & 229.72 & \pm 0.11 & 106.82 & \pm 0.18 & 0.37 & \pm 0.01 \\
$2.0 \mathrm{~g} / \mathrm{L}$ & 229.75 & \pm 0.10 & 106.84 & \pm 0.18 & 2.17 & \pm 0.01 \\
$5.0 \mathrm{~g} / \mathrm{L}$ & 229.79 & \pm 0.11 & 106.89 & \pm 0.18 & 5.18 & \pm 0.01 \\
\hline $0.1 \mathrm{~g} / \mathrm{L}$ & 117.42 & \pm 0.52 & 99.62 & \pm 0.39 & 4.69 & \pm 0.02 \\
$1.0 \mathrm{~g} / \mathrm{L}$ & 117.43 & \pm 0.52 & 99.63 & \pm 0.39 & 5.57 & \pm 0.02 \\
$5.0 \mathrm{~g} / \mathrm{L}$ & 117.49 & \pm 0.52 & 99.69 & \pm 0.39 & 9.49 & \pm 0.04 \\
\hline
\end{tabular}

\section{CONCLUSION}

We have evaluated the various operating modes of the CHKED software. The traditional analysis modes, identified in the software as "Classic," provide equivalent measurement results to that of the VMS-based HKED software. An MEKED approach to complex actinide solutions has been implemented in the CHKED software. The MEKED analysis provided reasonable results, but these results do not meet the International Target Values performance objectives for the HKED.

The MEKED method seems to work well for the traditional HKED solution ranges (e.g., $\mathrm{U}$ and $\mathrm{Pu}$ solutions containing little or no minor actinides concentrations and the U:Pu ratio is 100:1). The implementation of the MEKED algorithms allows determination of two major actinide components, typically $\mathrm{U}$ and $\mathrm{Pu}$, and one or more minor actinides (however, we were not able to obtain assay results for more than a single minor actinide). The CHKED treatment of the minor actinides appears erratic with a significant dependency of the assay result on the sample declaration.

The measurement precision achievable by the CHKED MEKED algorithms are consistent with the International Targets Values, but the overall measurement performance does not seem to meet these goals. This is partly because of the smaller diameter of the SGN vial compared to the path length of the glass cuvette (the target values were developed from a system utilizing the cuvettes) but also due to the limitations of the method. The presence of additional actinides limits the width of the ROIs, which limits the measurement precision. Reliance on a reference spectrum introduces an additional instrumental error component. Coding issues such as the apparent linkage of minor actinide declaration to the final analysis outcome may also degrade the performance for uranium and plutonium.

The CHKED MEKED method offers improved performance and flexibility compared to the VMS-based HKED analysis for the more complex actinide encountered in today's reprocessing facilities. However, the technique needs additional development. At a minimum, we recommend the following three simple modifications to the CHKED MEKED be implemented to partially address the development need.

- A pile up correction should be added to the analysis sequence. This will provide a more accurate determination of the background correction, and minimize the impact of variable $\mathrm{X}$-ray generator current settings (e.g. $5 \mathrm{~mA}$ vs $10 \mathrm{~mA}$ ).

- The method of subtraction of the continuum or stepped background remains unchanged from the traditional analysis approach. However, we have demonstrated that this method is incorrect and can lead to biases on the order of the KED target performance values (i.e., $0.3 \%$ or greater). It is recommended that the background correction methodology be updated to better reflect the true 
origin of the background counts. A correction to the lower ROI background region scaled to the ${ }^{109} \mathrm{Cd}$ peak rate should be sufficient to eliminate this source of error.

- The temperature correction developed for the $20 \mathrm{~mm}$ thick glass cuvette is applied to all KED measurements regardless of the sample container or method of temperature measurement (some facilities use the temperature of the hot cell rather than inside the HKED). Parameters used in the temperature correction should be made accessible to allow system/sample container specific corrections. 


\section{APPENDIX A}

\section{Impact of Background Subtraction on the K-Edge Densitometry (KED) Transmission Result}

The VMS Hybrid K-Edge Densitometer (HKED) ${ }^{2}$ and Windows-based CHKED ${ }^{3}$ software remove background from the KED spectra using a region of interest (ROI)-based correction technique. In this approach, there is assumed to be a rectangular tail (step background) associated with each gamma-ray or $\mathrm{X}$-ray peak. (Note: For the continuous energy distribution from the X-ray generator, each energy bin is treated as a peak.) The correction method makes use of two ROIs, one above and one below the energy region of interest, located in portions of the spectrum containing no peaks or other features. The calculated background as a function of energy in between these ROIs is based on the average count per channel in these two ROIs scaled by the ratio of the summation of counts in the spectrum between (1) the lower ROI and the current channel number and (2) the total number of counts in between the ROIs.

As we will demonstrate, the ROIs are not representative of the background continuum. The lower ROI includes tailing from the tungsten X-ray peaks and a significant contribution from the tail of the ${ }^{109} \mathrm{Cd}$ peak, and the upper ROI is populated by random coincidence summing events. The contents of these ROIs have little connection to the actual background continuum. In the following sections we explain the traditional approach and, then provide a more detailed physics-based description. This is used to provide a more meaningful and accurate representation.

\section{Step background}

The traditional HKED analysis described by Ottmar and Eberle [1] applies a "stepped background" correction to the measured transmission and XRF spectra. Figure 4 shows a common representation of the response of a $\mathrm{Ge}(\mathrm{Li})$ detector to a mono-energetic photon source. The continuum background shown in the figure is generally referred to as the step background.

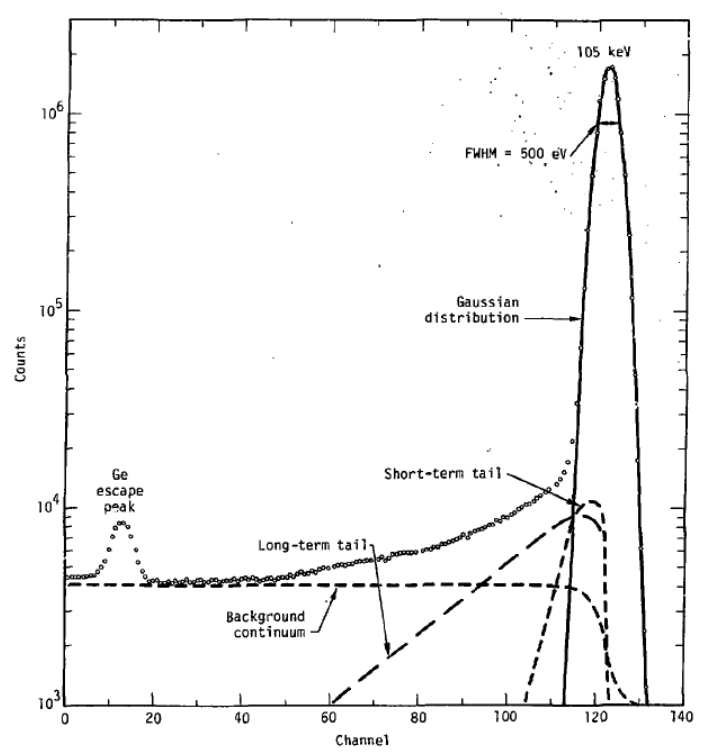

Figure 4. Peak shape from a Ge(Li) detector (from [5]).

\footnotetext{
${ }^{2}$ VMS Operating system based Hybrid K-edge Densitometry software, available from Canberra Industries

${ }^{3}$ Canberra Hybrid K-edge Densitometer Software, available from Canberra Industries
} 
Figure 5 shows the KED step background shape for a $321.9 \mathrm{~g} \mathrm{U} / \mathrm{L}$ solution as determined following the traditional HKED analysis (described in [1]). In the traditional HKED treatment, the magnitude of the step background for this spectrum is equivalent to $0.6 \%$ of the spectrum height just below the K-Edge transition and $2.5 \%$ of the spectrum height just above the U K-Edge.

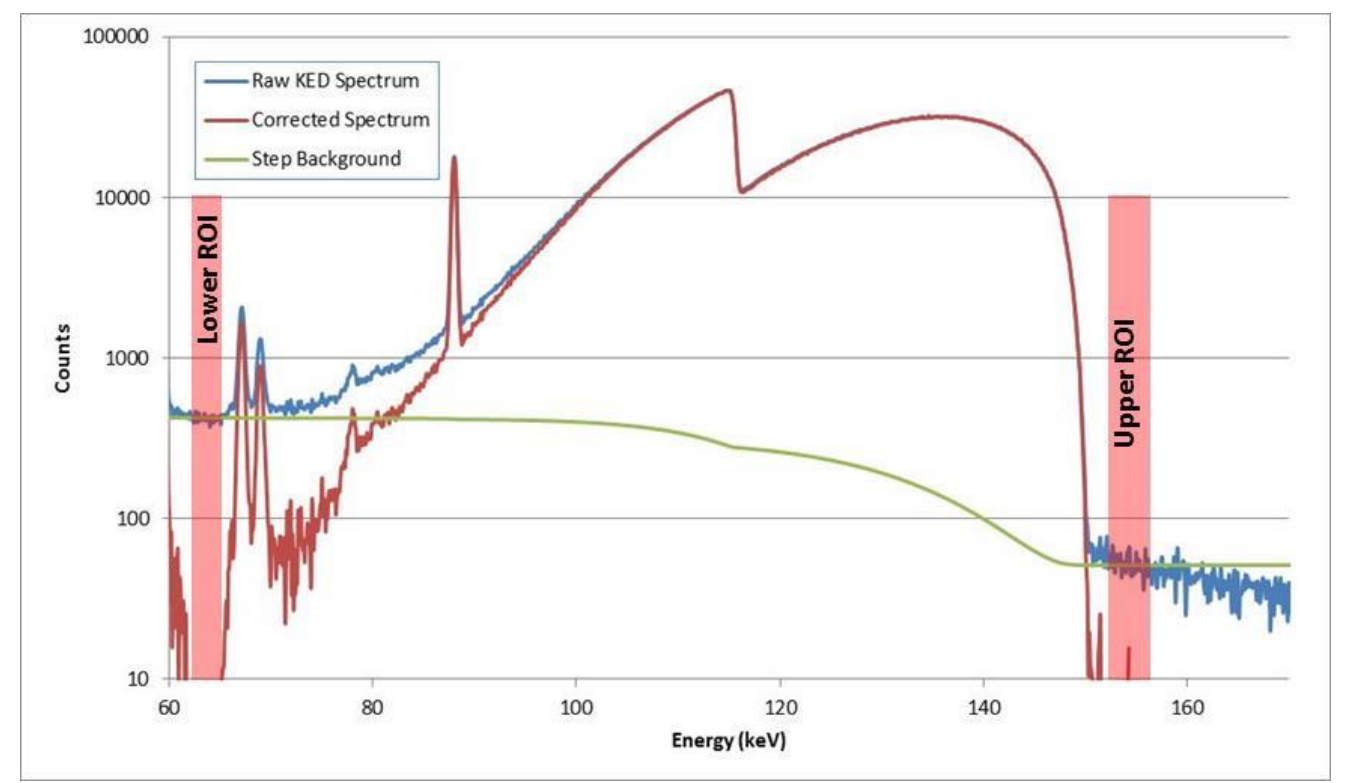

Figure 5. Step background determined for a $321.9 \mathrm{~g}$ U/L solution using the VMS and CHKED algorithm.

The step background, $I_{B K G}$, as a function of spectrum channel number shown in Figure 5 is described in Ref. [1] by the following expression

$$
I_{B K G}(n)=B_{L}+\left(B_{R}-B_{L}\right) \cdot \sum_{j=L}^{j=n} Y(j) / \sum_{k=L}^{k=R} Y(k),
$$

where $Y(i)=$ spectra counts of channel $i$,

$\mathrm{B}_{\mathrm{L}}$ is the average counts in the left background ROI (Figure 5),

$\mathrm{B}_{\mathrm{R}}$ is the average counts in the right background ROI (Figure 5).

The step background is a common feature in gamma-ray spectroscopy text books (e.g., Knoll's Radiation Detection and Measurement [6]) and scientific publications. However, there is little said about the origin of this step background or its properties. Most references eventually trace back to Gunnick's 1975, "Status of Plutonium Isotopic Measurements by Gamma-Ray Spectrometry" [7]. In this reference Gunnick attributes the step background to detector edge effects and small angle scattering by material between the detector and the source. However, the high degree of collimation used in the HKED detectors results in a pencil beam incident on a thick detector and eliminates these interferences - except for the ${ }^{109} \mathrm{Cd}$ lines and tungsten X-rays induced near the HPGe detector, which occur below the energy range of interest. In other words, the HKED analysis subtracts a background component that may not be present in the KED spectrum.

The following sections explore the source of counts in the background ROIs employed by the KED analysis. 


\section{Upper (right) background ROI}

For product materials (i.e., no fission products) the upper ROI should not have any counts in it. The observed counts are due to random coincidence summing (this has been verified by calculation and by varying the integrating X-ray beam intensity). More importantly, the HKED background subtraction model assumes that the background component is monotonically decreasing with increasing energy. The plot in Figure 6 clearly shows that this is not the case. Use of the upper ROI will lead to an oversubtraction of the background under the K-Edge transition.

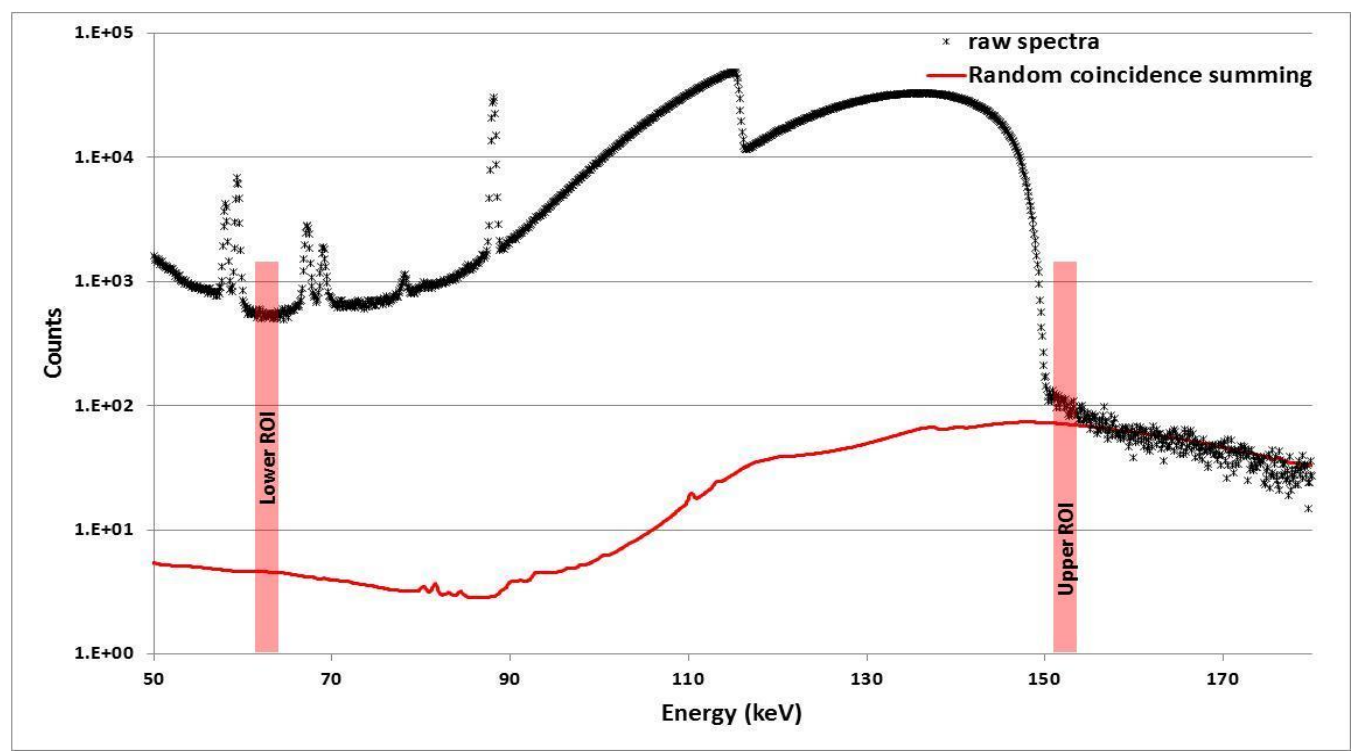

Figure 6. Plot showing the random coincidence summing contribution to the KED transmission spectrum. The ROIs (shaded area) are used in the VMS HKED and CHKED software for background correction.

\section{Lower (left) background ROI}

The lower background ROI cannot be described as a simple down scatter tailing from the transmitted Xray spectrum. The lower background ROI includes contributions from

- $\quad{ }^{109} \mathrm{Cd}$ source tailing,

- tungsten X-ray peak tails, and

- random coincidence summing.

Random coincidence summing-From Figure 6 above, we note that there is a small contribution to the lower ROI from random coincidence summing. Random coincidence summing contributes $1 \%-2 \%$ of the lower "background" ROI counts.

Cadmium-109 source tailing - The primary contribution to the lower (left) background ROI for product solutions is due to the tungsten X-ray fluorescence lines induced by the ${ }^{109} \mathrm{Cd}$ source bracketing the lower ROI as illustrated in Figure 7. The figure shows a KED detector spectrum acquired with the X-ray generator off and no sample in place. It should be noted that due to the location of the ${ }^{109} \mathrm{Cd}$ source within the tungsten shield, in close proximity to, but significantly off center from, the detector centerline, the $88.034 \mathrm{keV}$ peak shape is not representative of the detector response for photons emitted from the X-ray generator or the sample vial incident on the detector face in tight collimation. This is because the energy is deposited over a different region of charge collection electric field. The result is that there is a very large tail associated with the ${ }^{109} \mathrm{Cd}$ peak, and the Ge escape peaks are relatively more intense. For the Oak 
Ridge National Laboratory (ORNL) HKED system, the contribution to the lower ROI counts from the ${ }^{109} \mathrm{Cd}$ source is 0.024 times the ${ }^{109} \mathrm{Cd}$ peak intensity.

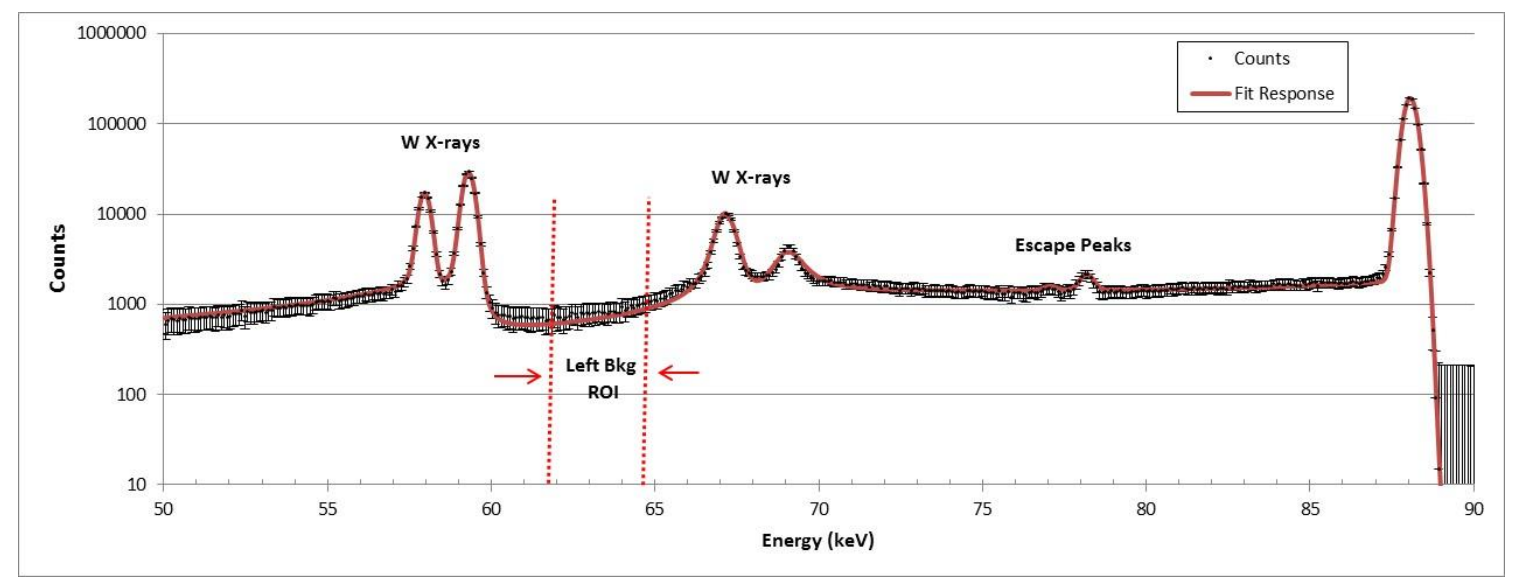

Figure 7. Plot of the passive (X-ray off) KED spectrum showing the contribution to the lower ROI from the ${ }^{109} \mathrm{Cd}$ source (acquisition time $=40,000 \mathrm{~s}$, peak rate $\sim 27 \mathrm{cps}$ ).

To examine the impact of the contributors to the lower ROI count rate, we acquired KED transmission spectra for the U300 uranium solution standard (321.9 g U/L) with and without the ${ }^{109} \mathrm{Cd}$ energy stabilization source (Figure 8). As can be seen in Figure 8 the tail extends through the lower background ROI.

Tungsten X-ray peak tails-We can also see in Figure 8 that due to the Voigt shape associated with the $\mathrm{X}$-ray peaks, the tungsten X-ray peaks contribute to the lower background ROI. These peaks contribute only about $1 \%$ of background ROI. We note that the majority of the tungsten X-rays detected in this spectrum were induced by the decay of the ${ }^{109} \mathrm{Cd}$ source so that the contribution to the background will change as the source decays $\left({ }^{109} \mathrm{Cd}\right.$ half-life $\sim 1.267$ years $)$. 


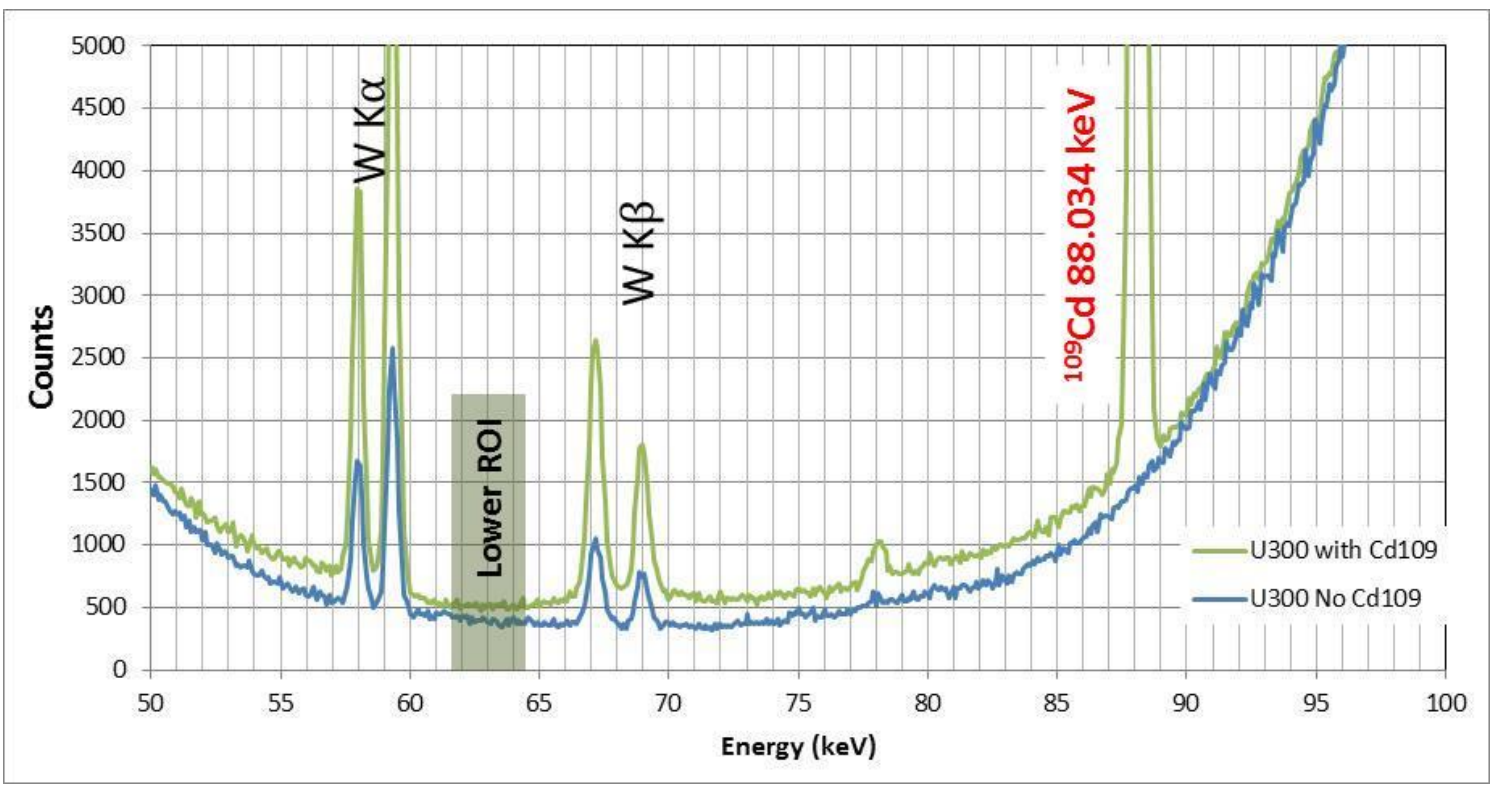

Figure 8. Measured KED transmission spectra illustrating the impact of the ${ }^{109} \mathrm{Cd}$ peak exponential tail on the lower background ROI. The large exponential tail from ${ }^{109} \mathrm{Cd}$ is due to its off-axis location in close proximity to the detector and surrounded by the tungsten shield. Count rate in the $88.034 \mathrm{keV}$ peak from ${ }^{109} \mathrm{Cd}$ was $153.5 \mathrm{cps}$. Note: Without the ${ }^{109} \mathrm{Cd}$ source, the count rate minimum is at $70 \mathrm{keV}$ confirming that the lower ROI includes counts other than the background.

For the spectrum shown in Figure 8, the X-ray generator was operating at $15 \mathrm{~mA}$ and $150 \mathrm{kV}$, and the ${ }^{109} \mathrm{Cd}$ peak count rate was $153 \mathrm{cps}$ and contributed $25 \%$ of the counts in the lower background ROI. Random coincidence summing contributed $1 \%$, and the tailing from the tungsten X-ray peaks contributed an additional $1 \%$ to the counts in the ROI. We estimate that in a typical assay the ${ }^{109} \mathrm{Cd}$ source contributes from $20 \%$ to $60 \%$ of the counts in the lower ROI depending upon the Cd source activity, X-ray tube current, and sample concentration. This contributor to the background ROI is time-dependent with the decay of the ${ }^{109} \mathrm{Cd}$ source.

Back scatter-For the $10 \mathrm{~mm}$ thick HPGe detector backscatter (from the copper cold finger) does not produce any significant count rate below $70 \mathrm{keV}$ and is not expected to impact the lower background ROI.

\section{OTHER CONTRIBUTIONS}

It is not clear that the remaining counts in the lower background ROI are attributable to a traditional stepped background. Figure 9 provides a comparison of a measured and MCNP [8] simulated KED spectrum. Comparison of the energy region between 70 and $75 \mathrm{keV}$ with the lower background ROI suggests an additional $5 \%$ of the lower background ROI is not related to a step background. We note in Figure 9 that in the energy region of $60-75 \mathrm{keV}$ the MCNP simulation severely underestimates the continuum underneath the tungsten X-ray peaks. This difference is in part attributable to the limitations of MCNP for the modeling of X-ray interactions; however, the very noticeable difference in the modeled and measured spectra suggests an additional contributor of unknown origin may exist. (Also note that because coincidence summing is not incorporated into the model, the tungsten $K_{\beta}$ line intensities are underestimated). 


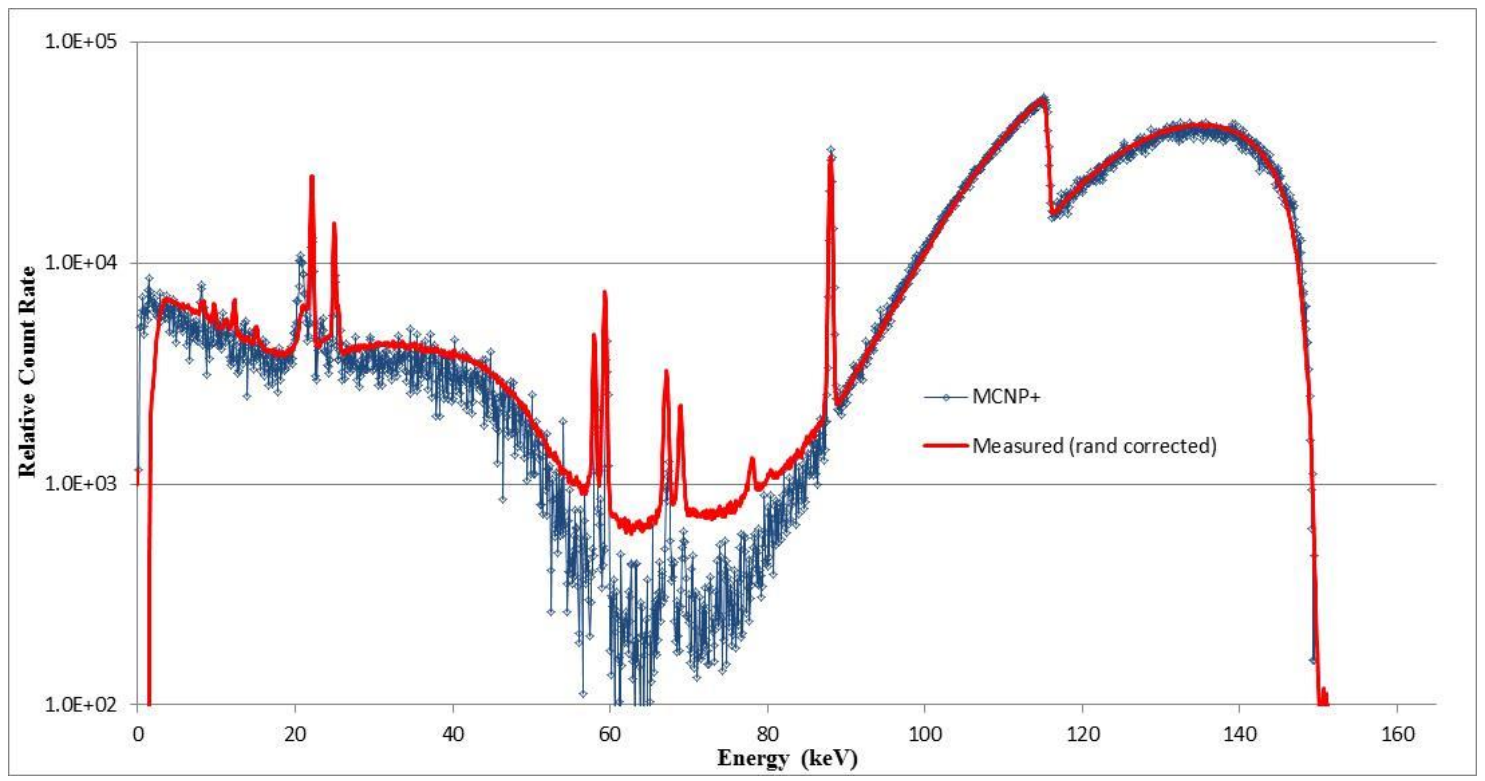

Figure 9. Comparison of the MCNP6 simulation of a KED transmission measurement for a $270 \mathrm{~g} \mathrm{U} / \mathrm{L}$ aqueous sample using corrected uranium photon cross-section libraries with a measured spectrum of the same concentration.

Because the scattering and tailing features of the ${ }^{109} \mathrm{Cd}$ peak are exaggerated compared to the transmitted $\mathrm{X}$-ray characteristic response, we conclude that current use of the lower background ROI significantly overestimates the background below the K-Edge transmission edge. We also note that the magnitude of the overestimation will change with time as the ${ }^{109} \mathrm{Cd}$ source decays.

As will be discussed, the empirical calibration of the KED measurement "hides" this bias to a large extent at the time of calibration, but as the ${ }^{109} \mathrm{Cd}$ source decays, a bias in the reported concentrations will develop.

\section{Representation of the Lower Background ROI Counts-Under Development}

We are presently investigating a more realistic representation of the background for the KED transmission measurement. Preliminary results based on a semiempirical model of the low energy portion of the KED spectrum are shown in Figure 10. Although the representation of the background is still under development, the results appear to confirm that almost all of the counts in the lower background ROI used in the existing CHKED analysis are due to tailing from the low energy scattering events unrelated to the step background. 


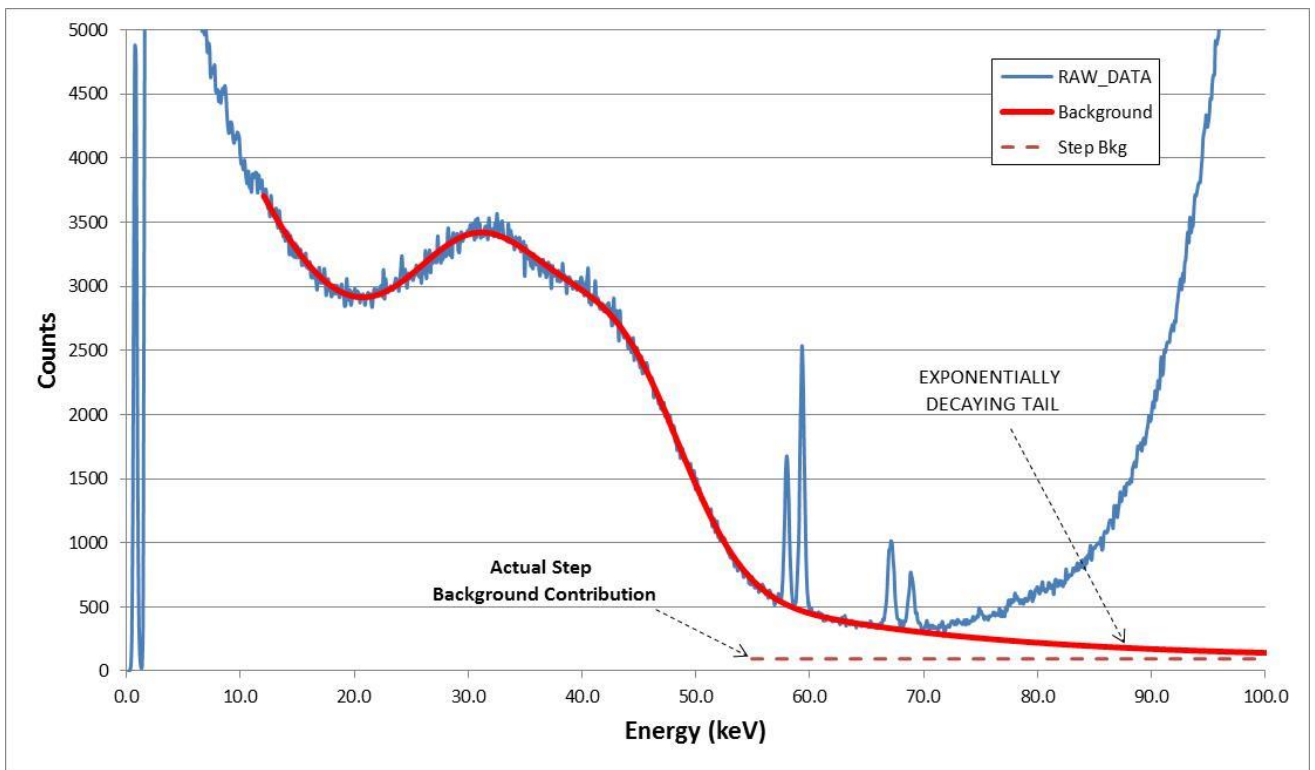

Figure 10. Low energy portion of the KED spectrum from a $321 \mathrm{~g} \mathrm{U} / \mathrm{L}$ sample without the ${ }^{109} \mathrm{Cd}$ source. The solid red line represents a semiempirical fit to the data.

\section{Impact of the ${ }^{109} \mathrm{Cd}$ Source on the Reported Uranium Concentration}

From the information available we cannot definitively state whether or not the step background contributes to the KED transmission spectrum; however, from the above discussions it is evident that the current HKED analysis (VMS and CHKED) overcorrects for this effect. In this section we examine the potential impact on the KED transmission results.

To investigate the impact of the decay of the ${ }^{109} \mathrm{Cd}$ source on the reported uranium concentration, a series of KED transmission spectra were created with increasing ${ }^{109} \mathrm{Cd}$ source contribution. Because a series of ${ }^{109} \mathrm{Cd}$ sources of sufficient activity were unavailable for this study, artificial spectra were produced by adding multiples of a measured passive ${ }^{109} \mathrm{Cd}$ spectrum to a measured KED transmission spectrum for a $322 \mathrm{~g}[\mathrm{U}] / \mathrm{L}$ sample. Although this approach lacks the subtle impact of increased random coincidence summing, it eliminates the complication of X-ray generator yield variation over the course of several assays. The resulting transmission spectra (Figure 11) provided effective ${ }^{109} \mathrm{Cd}$ peak rates up to $405 \mathrm{cps}$. 


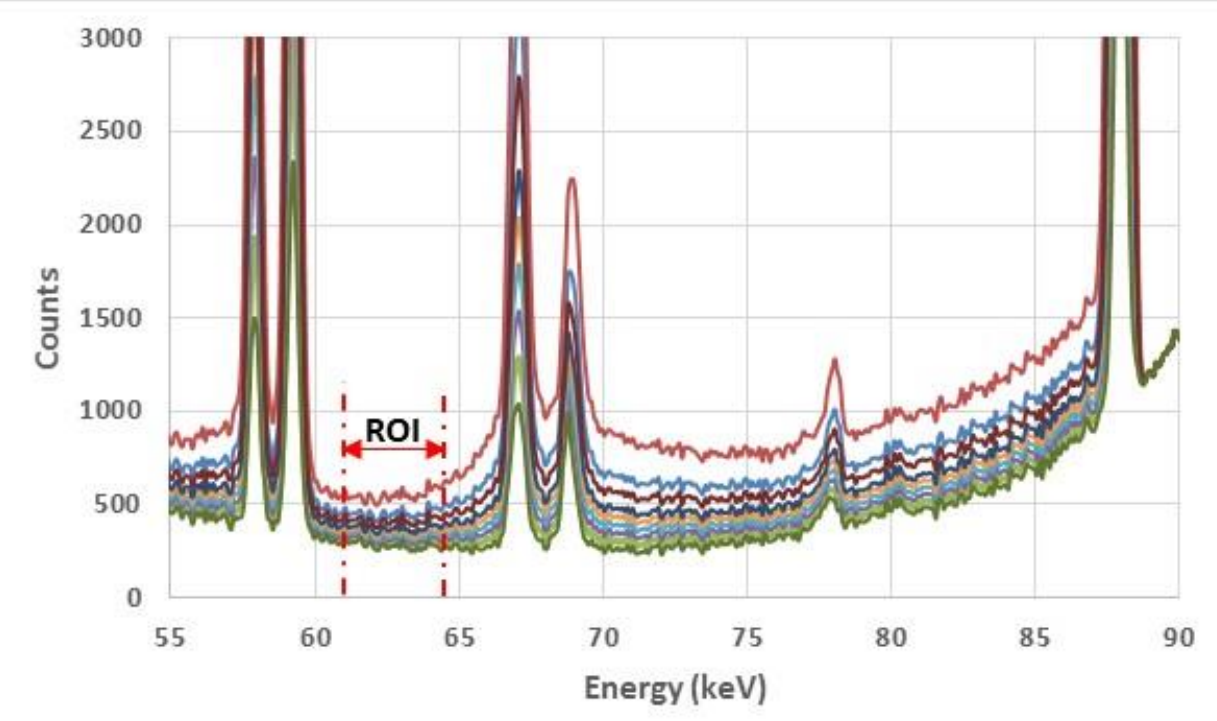

Figure 11. Plot illustrating the variation in the lower background ROI for the various ${ }^{109} \mathrm{Cd}$ source strengths.

The step background as a function of energy for each of these spectra was calculated in the manner described above. Figure 12 provides a plot of the step background count at and energy of $115.6 \mathrm{keV}$ at the K-Edge transition in uranium as a function of the ${ }^{109} \mathrm{Cd} 88 \mathrm{keV}$ peak count rate. The results are shown for KED spectra obtained at generator current settings of 10 and $15 \mathrm{~mA}$.

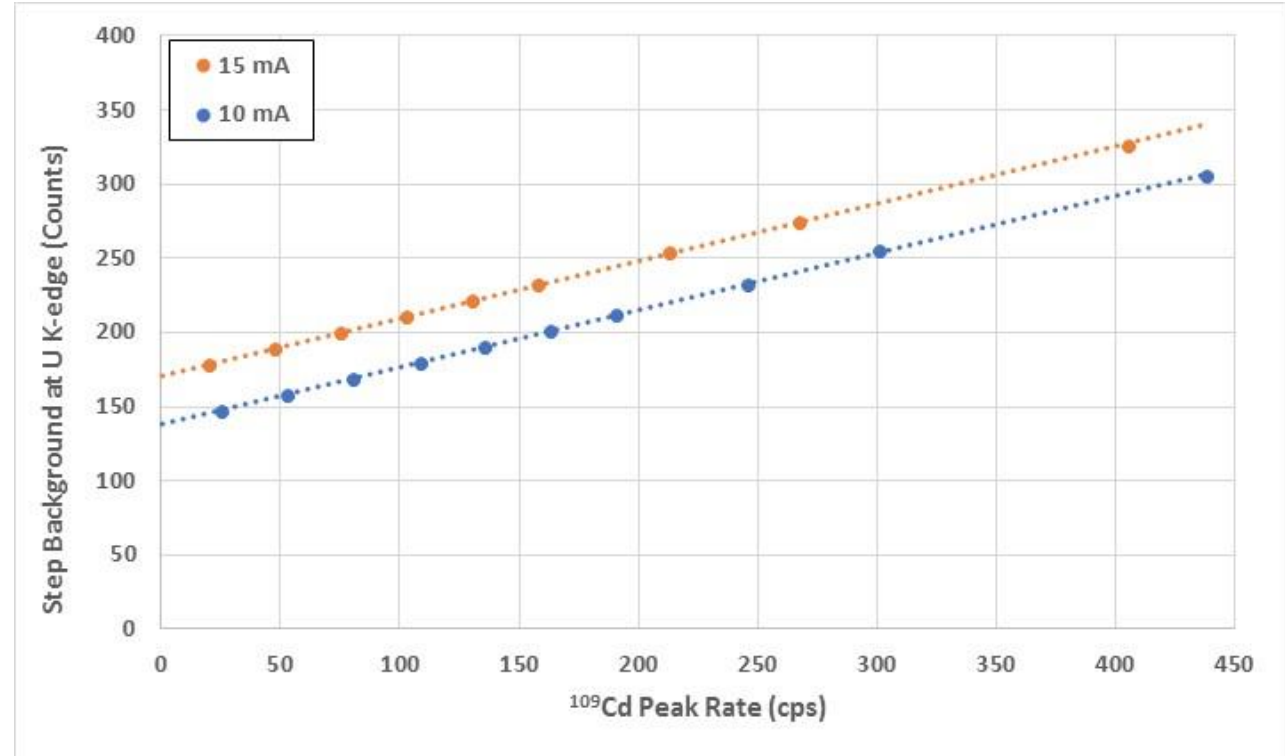

Figure 12. Plot of the calculated step background beneath the uranium K-Edge transition at $115.6 \mathrm{keV}$ for a 1,000 second assay of the $321 \mathrm{~g} \mathrm{U} / \mathrm{L}$ sample for two different current settings of the $\mathrm{X}$-ray generator.

To estimate the impact on the reported $[\mathrm{U}]$ concentration, a calibration was performed for the spectra with the largest ${ }^{109} \mathrm{Cd}$ peak rates for both the $10 \mathrm{~mA}$ and $15 \mathrm{~mA}$ current settings. The series of spectra were then analyzed using those calibrations to simulate the behavior of the system with a decaying ${ }^{109} \mathrm{Cd}$ reference source. The relative reported uranium concentration as a function of the ${ }^{109} \mathrm{Cd}$ rate is plotted in Figure 13 for a $322 \mathrm{~g}$ [U]/L sample. As the ${ }^{109} \mathrm{Cd}$ source decays, the step background counts subtracted 
from the raw KED transmission spectrum is reduced such that the transmission rate through the sample appears larger than at time of calibration resulting an underestimate (bias) of the uranium concentration.

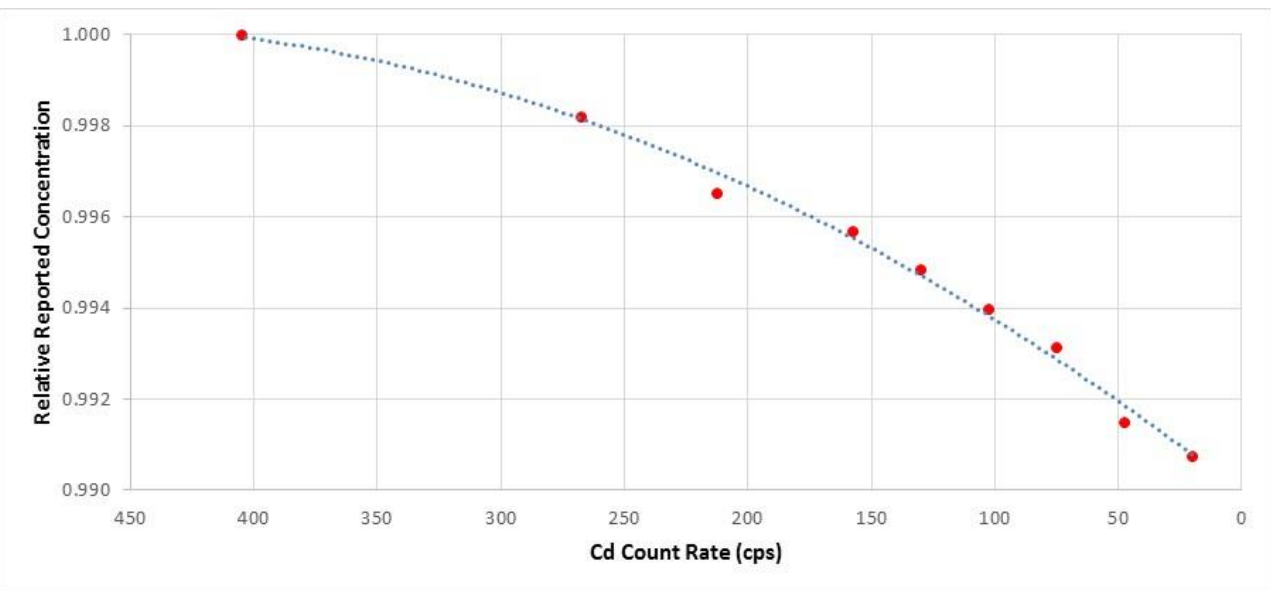

Figure 13. Relative reported $\mathrm{U}$ concentration of a $322 \mathrm{~g} \mathrm{U} / \mathrm{L}$ solution as a function of the ${ }^{109} \mathrm{Cd}$ peak count rate for the ORNL HKED system using an SGN vial with X-ray generator beam current of $15 \mathrm{~mA}$.

The data were replotted in Figure 14 as a function of time since calibration to illustrate the impact of the decay of the ${ }^{109} \mathrm{Cd}$ source. If the KED system is generally operated with a current setting of $15 \mathrm{~mA}$, the bias due to source decay is approximately $0.13 \%$ after 1 year, but if operated at $10 \mathrm{~mA}$, a bias of $0.25 \%$ can be expected after 1 year. To put this into perspective, the expected measurement precision for a $322 \mathrm{~g}$ $[\mathrm{U}] / \mathrm{L}$ sample in a typical assay is $0.2 \%$ so that the impact of the ${ }^{109} \mathrm{Cd}$ source decay is equivalent to the nominal measurement precision of the system. It should also be noted that the magnitude of the effect is impacted somewhat by the initial source rate as illustrated in Figure 15.

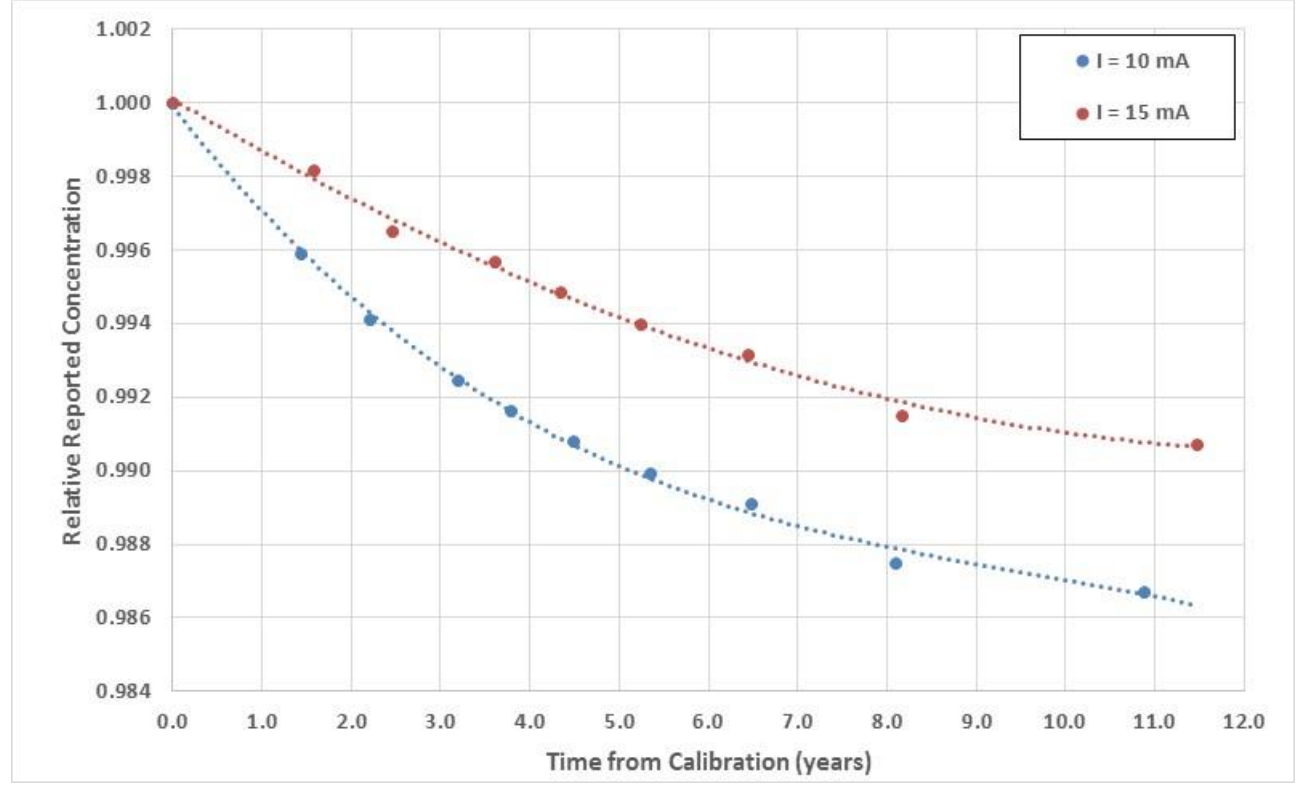

Figure 14. Relative reported concentration for a $322 \mathrm{~g} \mathrm{U} / \mathrm{L}$ sample as a function of time since last calibration assuming an initial Cd source rate of 420 cps. The impact of the Cd decay for X-ray beam intensities of 10 and $15 \mathrm{~mA}$ are shown for the ORNL HKED system using the SGN vial. 


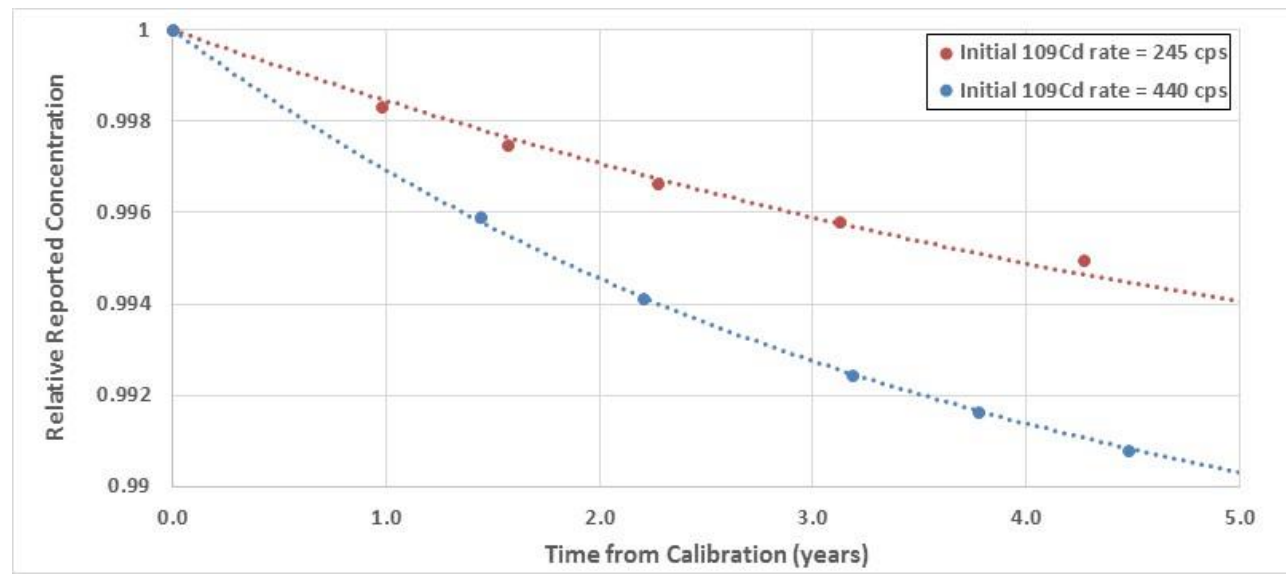

Figure 15. Relative reported concentration for a $322 \mathrm{~g} \mathrm{U} / \mathrm{L}$ sample as a function of time since last calibration assuming two different initial Cd source rates with $10 \mathrm{~mA} \mathrm{X}$-ray generator beam current using the ORNL HKED system using the SGN vial.

\section{SUMMARY}

For KED transmission analysis, the traditional correction based on the step background assumption does not accurately represent the properties of the KED background and overestimates the background that is subtracted from the KED spectrum. The KED transmission analysis implemented in the VMS and CHKED software accommodates this effect over the calibration range, and at the time of calibration, biases will not be evident. However, as the ${ }^{109} \mathrm{Cd}$ source decays, the fractional count rate in the lower background ROI changes relative to the transmitted spectrum, resulting in a bias that increases with both time and concentration. These biases, although small $(\sim 0.2 \%-0.5 \%)$ are significant compared to the KED measurement precision, accuracy, and performance target values. To minimize this effect, the KED transmission measurement must be recalibrated at 6-12 month intervals. Alternatively, a correction to the lower background ROI based on the observed count rate in the ${ }^{109} \mathrm{Cd}$ peak could be applied. We have not yet evaluated the impact of the background correction on plutonium assay or mixed actinide (e.g., $\mathrm{U}=\mathrm{Pu}$ solutions). Although the impact on the plutonium assay is expected to be less severe because the background decreases at higher energies.

As can be seen in Figure 8, the count rate in the KED spectrum without ${ }^{109} \mathrm{Cd}$ present reaches a minimum at approximately $70 \mathrm{keV}$. So even without any contribution from ${ }^{109} \mathrm{Cd}$, the current $61.75-64.75 \mathrm{keV}$ window overstates the background correction. The count rate from the ${ }^{109} \mathrm{Cd}$ peak increases this overstatement. This is in part due to the higher energy Compton scattered events extending into the background ROI and the lower energy tail of the transmitted Bremsstrahlung spectrum through the steel beam filter. We have not, in this discussion, addressed the "correct" description of the background and only point out that the description used by the commercial software packages is incorrect and will introduce biases in the assay result in the absence of frequent recalibrations. 


\section{APPENDIX B}

\section{Examination of the Temperature Dependence of the ORNL HKED}

The temperature dependence of the K-Edge densitometry (KED) measurement described by Ottmar et al. [1] was determined for samples within a $20 \mathrm{~mm}$ long rectangular glass cuvette, whereas the sample vial used with the Oak Ridge National Laboratory (ORNL) system is a $14.18 \mathrm{~mm}$ diameter, high-density polyethylene, cylindrical vial. To examine the differences in the temperature dependencies for the two sample containers a series of measurements were performed with ORNL system. For these measurements a thermocouple was placed inside the protective lead shield but just outside HKED sample transfer tube. A second thermal couple was located outside of the shield housing (Figure 16). Eighteen 1,000 second repeat measurements were performed on a $321 \mathrm{~g} \mathrm{U} / \mathrm{L}$ sample and the reported concentrations presented in Figure 17. The measurements were conducted consecutively, and the system temperature increased smoothly throughout these measurements because of heating by operation of the $\mathrm{X}$-ray generator. These concentrations include the CHKED correction factor of $0.05 \% /{ }^{\circ} \mathrm{C}$ and result in a bias of $0.11 \% /{ }^{\circ} \mathrm{C}$. Undoing the temperature applied by the software, Figure 18 shows that the temperature correction applied by the software increases the reported bias rather than eliminating it. We find that the temperature correction for the ORNL system using the SGN vials is

$$
\rho_{25}=[1-0.0006 \cdot(T-25)] \cdot \rho_{T},
$$

where $\rho_{25}$ is the concentration corrected to $25^{\circ} \mathrm{C}$,

$\rho_{T}$ is the concentration at the measurement temperature,

$\mathrm{T}$ is the temperature of the "sample transfer tube" during measurement.

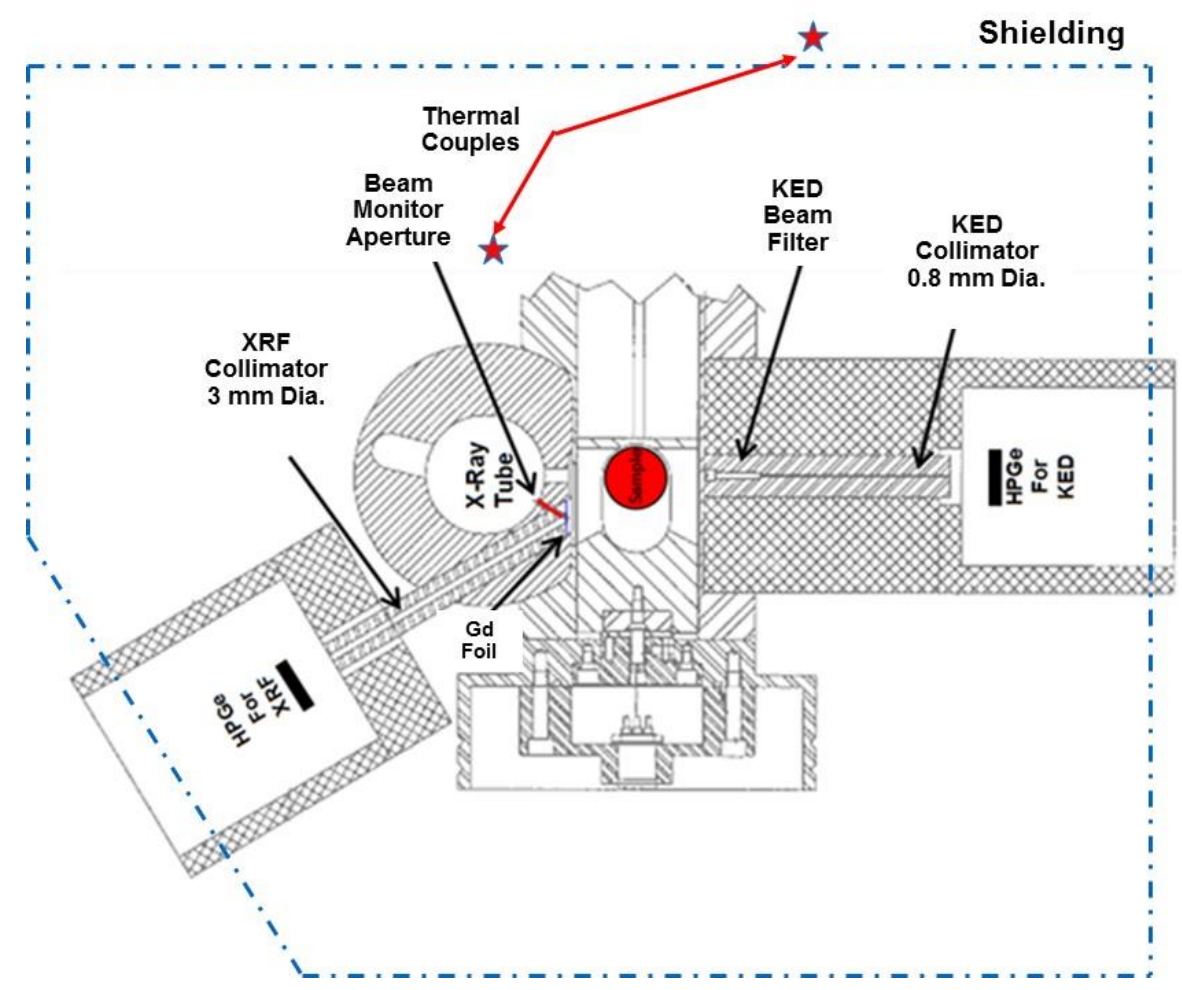

Figure 16. Sketch of the ORNL HKED system showing the approximate location within the shield housing of the thermal couple used for sample temperature measurement. 


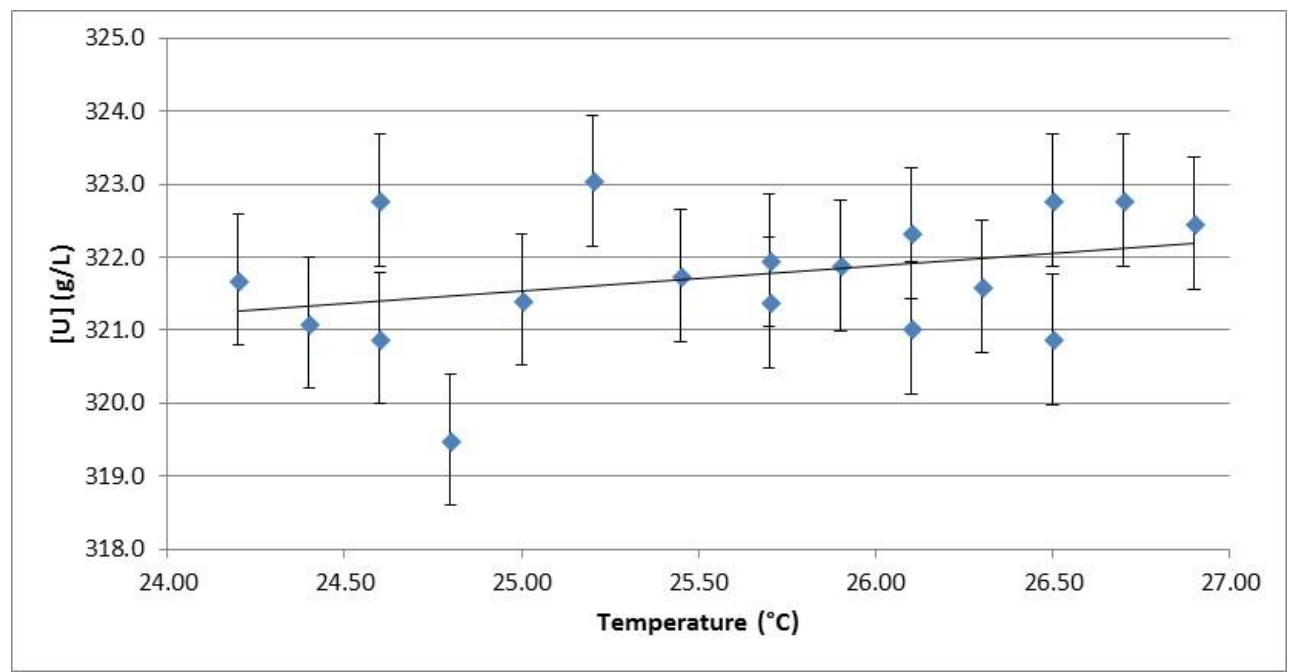

Figure 17. Concentration reported by the CHKED software as a function of temperature showing a temperature dependence of $+0.11 \% /{ }^{\circ} \mathrm{C}$.

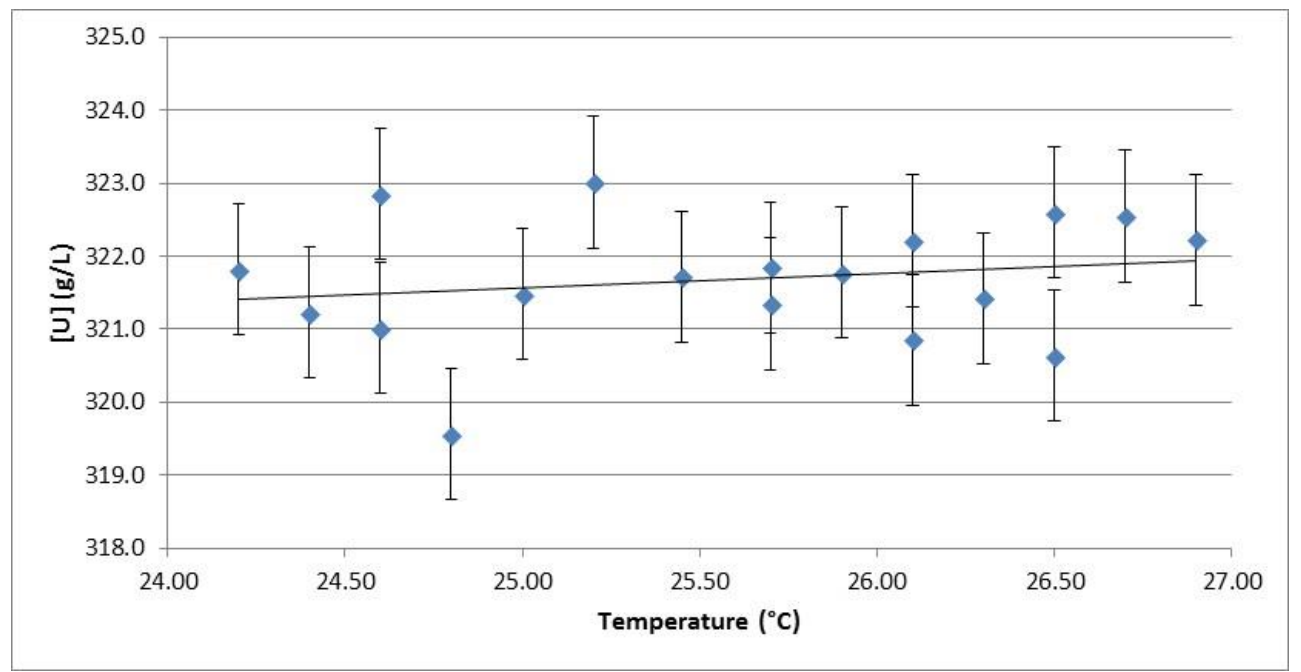

Figure 18. Concentration reported by the CHKED software as a function of temperature after the temperature correction has been removed and exhibiting a temperature dependence of $+0.06 \% /{ }^{\circ} \mathrm{C}$.

However, because the reported concentration appears to increase with temperature rather than decrease as expected, the uncorrected concentration values for the data acquired and analyzed with the CHKED software were compared with similar series of measurements performed using the VMS HKED software (Figure 19). Data obtained using the VMS software behaves more as expected and yields a temperature correction that is larger in magnitude than that reported for the glass cuvettes in Ref. [1].

$$
\rho_{25}=[1+0.002 \cdot(T-25)] \cdot \rho_{T}
$$

We have no explanation for the rather puzzling behavior of the CHKED results. 


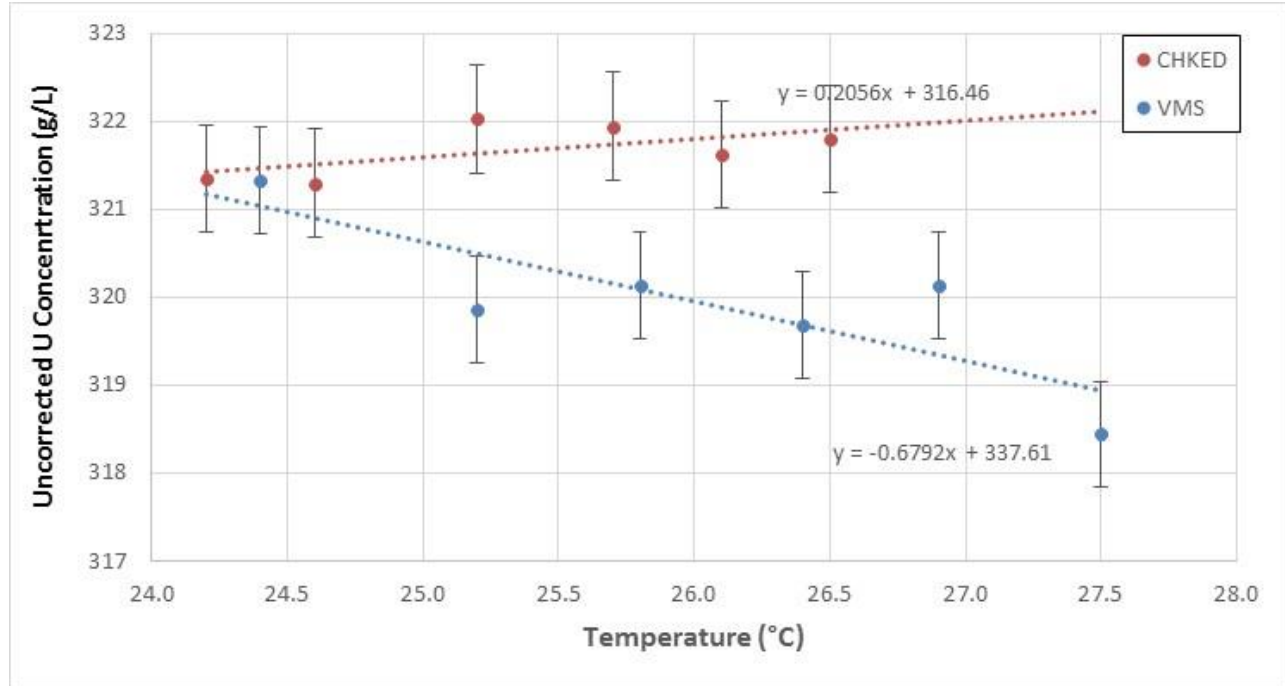

Figure 19. Comparison of the uncorrected uranium concentration as a function of temperature analyzed using the VMS KED analysis and the CHKED analysis. 


\section{APPENDIX C}

\section{Sample Vial Considerations}

Historically, the Hybrid K-Edge Densitometer (HKED) systems used separate sample vials for the KEdge densitometry (KED) and X-ray fluorescence (XRF) measurements both contained within an outer carrier. Four HKED systems installed at the AREVA facilities in La Hague were later designed for use with a pneumatic transfer system that would accommodate only a simple single sample vial configuration. This single vial concept was adopted as the standard configuration for its simplification of sample handling. However, the optimal sample size is dependent upon the actinide and matrix composition and density. The HKED systems for aqueous process solutions were optimized for KED transmission measurements where $100<[\mathrm{U}+\mathrm{Pu}]<400 \mathrm{~g} / \mathrm{L}$. The downside of this configuration is that the sample vials are larger and require more solution than otherwise needed.

The larger sample volume is detrimental in terms of increased gamma-ray exposures, larger waste volumes generated, and increased interferences impacting the assay results (e.g., increased self-florescence effects). Although the impact of sample dimensions on the KED measurement is rather straightforward, evaluation of the impact on the XRF measurement is significantly more complex. We initially employed MCNP simulations to examine the interaction region of the X-ray beam within the sample volume to develop improved conversion factors for the XRF U:Pu determination [9] [10] [11], and it was readily apparent that the sample volume contributing to the XRF and KED measurement result was only a small fraction of the total sample volume.

The simulations used to better describe the extent of the detectable XRF interaction region are shown in (Figure 20 and Figure 21). These show the sub-volume of the sample where K-shell vacancies are created by the interrogating X-ray beam resulting in the emission of characteristic X-rays that can be subsequently detected. These simulations allowed us to refine the XRF analysis algorithms (e.g., improved treatment of the attenuation and transmission corrections) and can be used to aid in the design of an optimum sample vial. For instance, the heat map shown in Figure 21 illustrates that the sample volume necessary for the XRF analysis may be as small as $4 \times 3 \mathrm{~mm}(\mathrm{D} \times \mathrm{H})$ or less than $1 \%$ of the current $5 \mathrm{~mL}$ sample volume. 

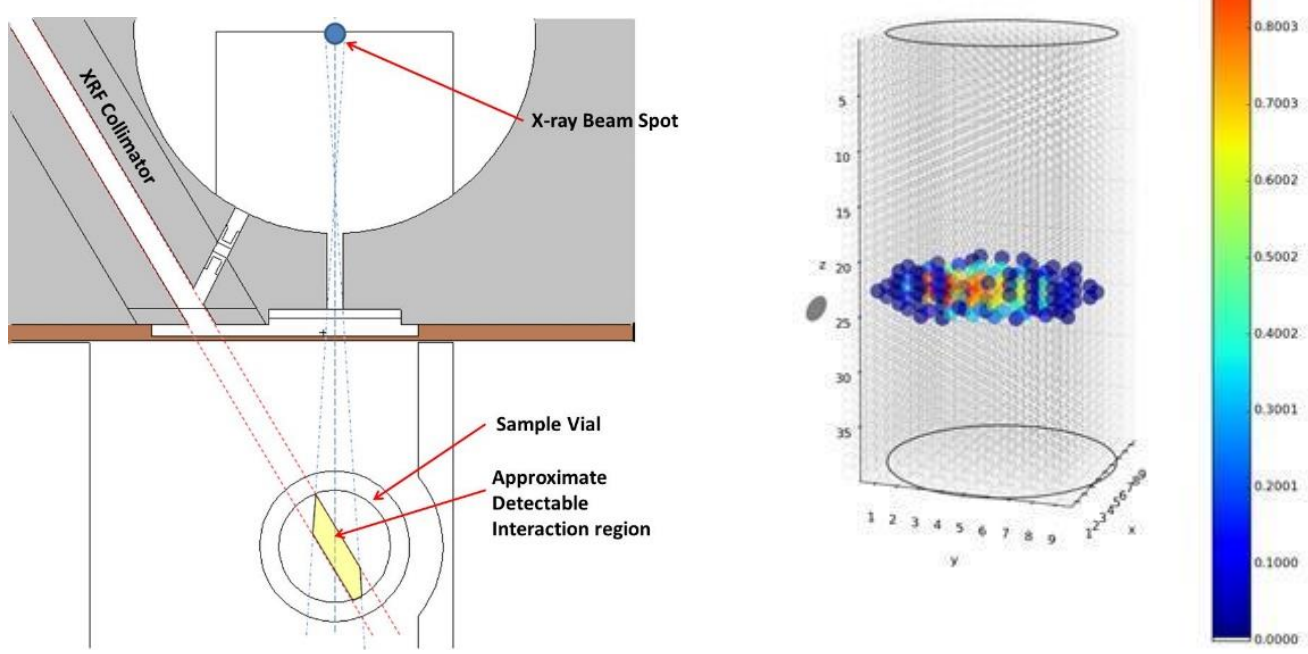

Figure 20. Determination of the XRF-detectable interaction region by MCNP simulation. The drawing at left is a simple line drawing estimate of the active region. The drawing at right illustrates the simulated interaction probabilities as a function of position within the vial showing that the interaction volume is significantly smaller than original estimates [9].
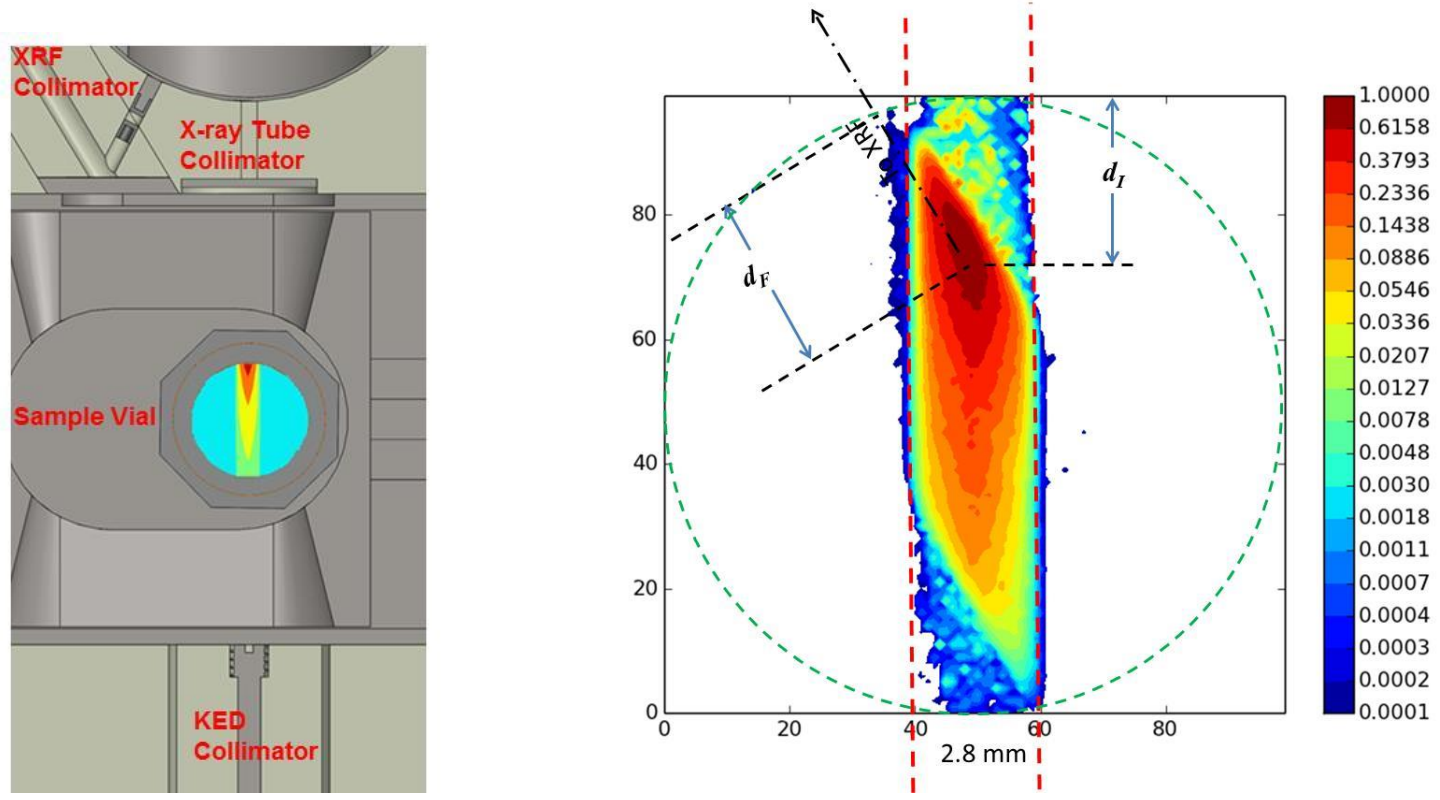

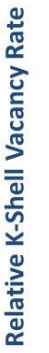

Figure 21. Heat map of the XRF-detectable interaction region determined by MCNP simulation. This figure [9], generated from MCNP6 simulations, shows the effective interaction region within a $250 \mathrm{~g} \mathrm{U} / \mathrm{L}$ in $3 \mathrm{M}$ nitric acid solution leading to detectable fluorescence X-rays (note the log scale). The circle represents the inner diameter of the sample vial. Even though interrogating X-ray beam has spread from $2 \mathrm{~mm}$ to $2.8 \mathrm{~mm}$ wide due to scattering at the tungsten aperture, $\mathrm{K}$-shell vacancy production is essentially confined to a narrow region of the sample vial.

The oversized sample volume does not simply waste solutions, but it also introduces two main interferences: 
1. The XRF measurement the fluoresced actinide $X$-rays are attenuated as pass through the inactive portion (the portion not in the transmission beam) of the sample volume causing loss of signal and increased non-linearity in response.

2. Solution outside of the interaction region does not beneficially contribute to the XRF spectrum however fission products within the entire sample volume contribute to self-fluorescence.

The heat maps suggest that a single rectangular sample vial similar to the original glass cuvette without the separate high-density polyethylene would adequately enclose the necessary sample volume. But keeping in mind the dimensions of the collimating apertures used with the HKED, the maximum fill height required is less than $5 \mathrm{~mm}$. The simple evaluation suggests that a rectangular sample container measuring $15 \times 3 \times 5 \mathrm{~mm}(\mathrm{~L} \times \mathrm{W} \times \mathrm{H})$ and containing less than $0.25 \mathrm{~mL}$ of solution could be positioned within the existing SGN-style HKED system with minimal modifications, while providing equivalent or superior results to the current $5 \mathrm{~mL}$ sample vial. 


\section{REFERENCES}

[1] H. Ottmar and H. Eberle, "The Hybrid K-Edge/K-XRF Densitometer: Principles - Design Performance," Report no. KfK 4590, Karlsruhe, 1991.

[2] R. D. McElroy, "Performance Evaluation of the CHKED Multi-Elemental Analysis Software," Oak Ridge National Laboratory, Oak Ridge, TN, 2016.

[3] H. Eberle and H. Ottmar, "TT Bericht Windows Based Calibration of the Hybrid K-Edge / XRF Spectrometer," Institut for Kernphysik III, Kernforschungszentrum Karlsruhe GmbH, Karlsruhe, Germany, 1993.

[4] Zhao, K. et.al., "International Target Values 2010 for Measurement Uncertainties in Safeguarding Nuclear Materials, Report No. STR-368," International Atomic Energy Agency, Vienna, November 2010.

[5] R. Gunnick and J. B. Niday, "Precise Analyses by Gamma-Ray Spectroscopy," in ACS Annual Meeting, August 24-29 1975, Chicago, 1975.

[6] G. F. Knoll, Radiation Detection and Measurement, New York: John Wiley and Sons, 1979.

[7] R. Gunnink, "UCRL-76418 Status of Plutonium Isotopic Measurements by Gamma-Ray Spectrometry," in American Nuclear Society 21 st Annual meeting, New Orleans, 1975.

[8] J. T. Goorley and et.al., "Initial MCNP6 Release Overview - MCNP6 version 1.0, LA-UR-1322934," Los Alamos National Laboratory, Los Alamos, NM, 2013.

[9] R. D. McElroy, Jr. and G. S. Mickum, "Application of Hybrid K-Edge Densitometry to International Safeguards in Support of Pyroprocessing Activities - Project Update, ORNL/TM2015/025," Oak Ridge National Laboratory, Oak Ridge, TN, 2015.

[10] R. D. McElroy, S. L. Cleveland, S. Croft, G. S. Mickum and A. Nicholson, "Relative Actinide KShell Vacancy Production Rates in Hybrid K-Edge Densitometry," in 37th ESARDA symposium on Safeguards and Nuclear Non-Proliferation, Manchester, UK, 19-21 May 2015.

[11] R. D. McElroy, S. Croft, G. S. Mickum and S. L. Cleveland, "Spectral Fitting Approach to the Hybrid K-Edge Densitometer: Preliminary Performance Results," in 56th Annual Meeting of the Institute of Nuclear Materials Management (INMM 56) (Proc. Conf. Indian Wells, CA, 2015), Curran Associates, Inc., Indian Wells, CA, 2015. 
